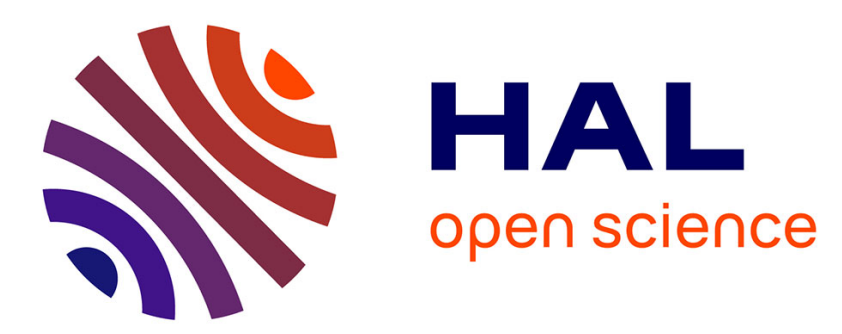

\title{
MRI-Guided Nanorobotic Systems for Therapeutic and Diagnostic Applications
}

Panagiotis Vartholomeos, Matthieu Fruchard, Antoine Ferreira, Constantinos

Mavroidis

\section{- To cite this version:}

Panagiotis Vartholomeos, Matthieu Fruchard, Antoine Ferreira, Constantinos Mavroidis. MRI-Guided Nanorobotic Systems for Therapeutic and Diagnostic Applications. Annual Review of Biomedical Engineering, 2011, 13, pp 157-184. 10.1146/annurev-bioeng-071910-124724 . hal-00647919

\section{HAL Id: hal-00647919 https://hal.science/hal-00647919}

Submitted on 5 Dec 2011

HAL is a multi-disciplinary open access archive for the deposit and dissemination of scientific research documents, whether they are published or not. The documents may come from teaching and research institutions in France or abroad, or from public or private research centers.
L'archive ouverte pluridisciplinaire HAL, est destinée au dépôt et à la diffusion de documents scientifiques de niveau recherche, publiés ou non, émanant des établissements d'enseignement et de recherche français ou étrangers, des laboratoires publics ou privés. 


\section{MRI-guided nanorobotic systems for therapeutic and diagnostic applications}

Panagiotis Vartholomeos ${ }^{1,2}$, Matthieu Fruchard ${ }^{3}$, Antoine Ferreira ${ }^{3,}$, and Constantinos Mavroidis ${ }^{4, *}$

${ }^{1}$ Brigham and Women's Hospital, Harvard Medical School, Boston, MA, USA

<pvarthol@bwh.harvard.edu>

${ }^{2}$ Zenon Automation Technologies, Glyka Nera, Athens Greece

${ }^{3}$ Institut PRISME, University of Orleans, 18000, Bourges, France

<matthieu.fruchard@bourges.univ-orleans.fr>, <antoine.ferreira@ensi-bourges.fr>

${ }^{4}$ Bionano Robotics Laboratory, Dept. of Mechanical \& Industrial Engineering,

Northeastern University, Boston, MA, USA

<mavro@coe.neu.edu>; http://www.bionano.neu.edu

* Authors for Correspondence 


\section{Table of Contents}

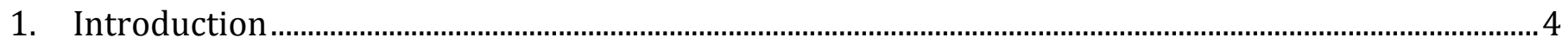

2. Concept of Controlled Drug Delivery Systems ……………..................................................................................

3. Overview of MRI-DDS System Architecture....................................................................................................

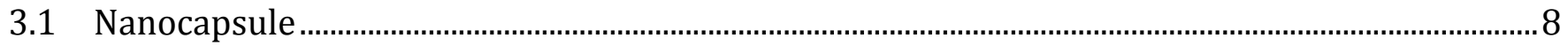

3.2 MRI Propulsion Unit .........................................................................................................................................

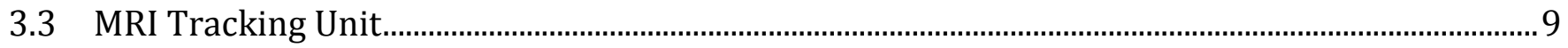

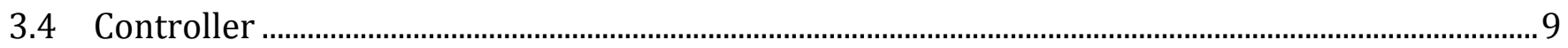

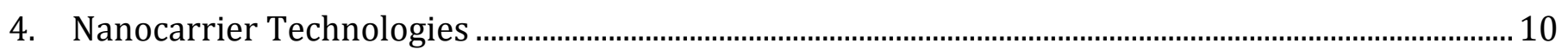

4.1 Liposome Nanoparticles ........................................................................................................ 11

4.2 Polymer Micelles ..........................................................................................................................................

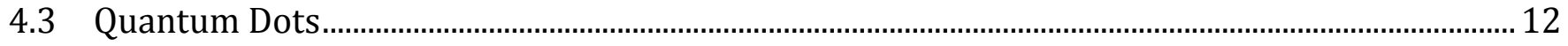

4.4 Metallic Nanoshells ................................................................................................................................... 12

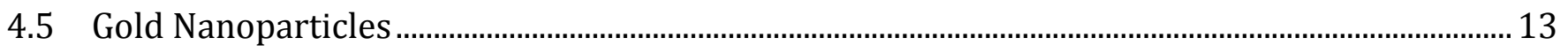

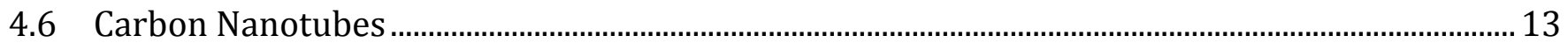

5. Nanoparticle functionalization for sensing purposes...............................................................................13

5.1 Targeting mechanism: Functionalization with biomolecules that act as "targeting

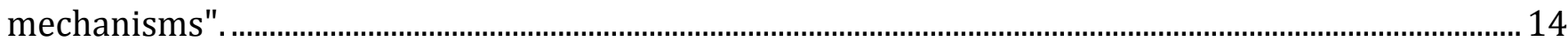

5.2 Mechanisms for evading immunogenic reactions: Functionalization of nanocarriers with

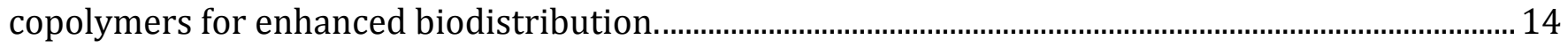

5.3 Triggered-release mechanisms: Functionalization of nanocarriers with copolymers

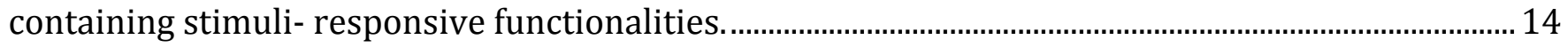

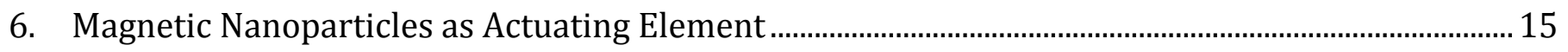

7. MRI Based Propulsion, Tracking and Control ................................................................................................ 17

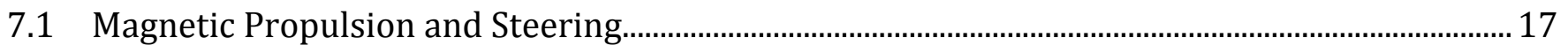

7.2 Imaging and Tracking in MRI Scanners............................................................................................... 19

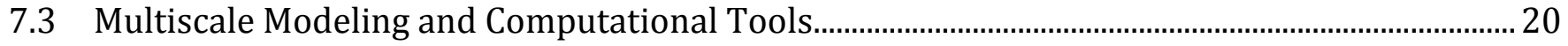

7.4 Control Algorithms for Navigation in MRI Environment.................................................................. 21

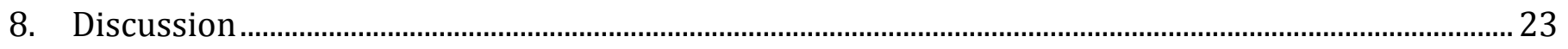




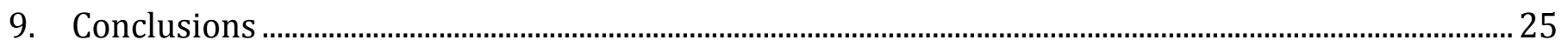

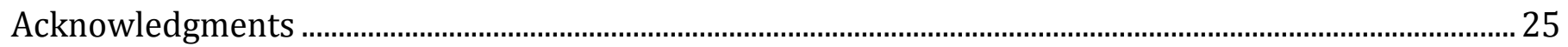

References

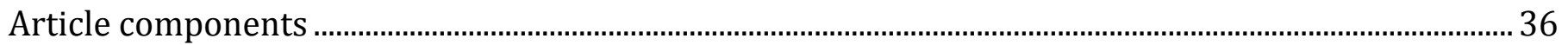




\section{Introduction}

Nanorobotic drug delivery systems guided by Magnetic Resonance Imaging (MRI) scanners have been proposed for targeted drug delivery in the human body. The expectation is that they will achieve substantially increased rates of therapeutic and diagnostic success compared to conventional methods. In this paper we present the state of the art in this relatively recent field of study.

Nanorobots are defined as controllable machines at the nanometer scale that are composed of nano-scale components and algorithmically respond to input forces and information (1). Nanorobotics for medical applications have been inspired by the function of antibodies, i.e. the natural organisms that our body uses to kill or repair diseased cells. Because of the comparable size with human cells, nanoparticles are the fundamental structure of the nanorobotic concept. Studies have shown that only objects of the size of $30-300 \mathrm{~nm}$ can be circulated through the thinnest sections of the vasculature system and would be capable of interacting with cells, (2-4). Furthermore, if the nanorobots acquire simple functionalities to sense and target specific cells, and to release drug molecules upon reception of a triggering signal, then a radically new approach in medicine would be realizable: to conduct curative and reconstructive treatment in the human body at the cellular and subcellular level in a controllable manner. Such a groundbreaking advancement would signal a new era in medical diagnosis and treatment of neoplasms, hepatitis, diabetes and other diseases, where highly-controlled and targeted treatment is critical for the survival of the patients. Such nanorobotic devices will hopefully be part of the arsenal of future medical devices and instruments that will: (1) perform operations, inspections, and treatments of diseases inside the body, and (2) achieve ultra-high accuracy and localization in drug delivery, thus minimizing side effects.

Over the last two decades a variety of nanoparticles and nanovesicle technologies have been developed. Many of these have been employed for diagnostic and therapeutic purposes (5-12). These novel nanoscale platforms possess excellent physiochemical properties, have prolonged circulation times and improved absorption rates, but are circulated systemically and thus exhibit poor targeting capabilities $(8,13)$. Enhancing 
the targeting capabilities of the nanoparticles and increasing their diagnostic and therapeutic efficacy necessitates the use of guidance techniques. This in turn, calls for the integration of sensing and actuation mechanisms on the nanocarrier. For this purpose, several groups have been studying and developing magnetic micro/nanoparticles (14-17) and have designed techniques for their controlled locomotion from an initial point to a desired final point in the vasculature. The research in this field was initiated in the early 2000 and initially focused on guided magnetic microparticles captured by a static magnetic fields generated by magnets (18-20) or superconducting magnets $(21),(22)$. In these studies the particles were simply attracted towards the targeted region without any tracking and feedback mechanism. This open loop approach resulted in a 75-fold higher concentration compared to conventional systemic administration. However, the static magnet limited efficient targeting only in regions found near the surface of the body and offered poor localization accuracy.

The next step was to attain a more precise targeting by developing a closed-loop nanoparticle localization system. To this end, in the past five years, two approaches of pulling magnetic micro and nanoparticles have been followed. The first is the use of external Maxwell coils (23-25) and the second is the use of an MRI device (26-30) to guide the nanocapsules. Another approach that has also been adopted is the use of magnetic implants $(31,32)$, however, this research is at its infancy, and the results are mostly limited to simulation studies. Furthermore, its implementation is hindered by the fact that it involves implants. Finally, magnetic propulsion techniques other than pulling micro and nanocapsules have also been proposed in the past five years. The most characteristics are (i) the biomimetic robots using flagella (33-35) and (ii) magnetotactic bacteria $(36,37)$.

The MRI approach is favored due to the fact that it is a non-invasive method, it allows for simultaneous actuation and tracking of the nanoparticles, it is capable of providing very accurate localization of the magnetic particles, and commercial MRI devices are readily available at most hospitals. Research on this field has been pioneered since 2003 by Sylvain Martel at the Ecole Polytechnique of Montréal $(26,27,29,38)$. He has used clinical MRI to navigate a $10.9 \mu \mathrm{m}$ magnetic microparticles in-flow into a branch of a Y- 
shaped microchannel (29). His analytical and experimental research results have been limited to milli and micrometer size non-functionalized magnetic particles. In (39) preliminary experiments were conducted, where a non-medical MRI was used to move and track micron size agglomerates composed of nanoparticles. No studies have been conducted so far on nanocarriers, whose motion is closed-loop controlled, and that have been functionalized for diagnostic or therapeutic purposes.

A systematic approach towards MRI-based guidance of nanoscale functionalized robotic capsules began for the first time, in the summer of 2008 in the context of the European Project NANOMA (http://nanoma.zenon.gr). The innovative concept of MRI-guided nanorobotic systems is to use an MRI scanner to apply to the nanoparticles an external driving force to guide them and retain them at a localized target. The direction and magnitude of the forces applied on the nanoparticles are generated according to a control law, whose feedback - the endovascular position of the nanoparticles - is provided by processing the MRI data. Navigation techniques in combination with appropriate chemical modification of the nanoparticles surface yield a more localized and controlled treatment as well as controlled drug-release mechanisms.

Many diverse technologies and disciplines are involved in the development of an MRI-based nanorobotic drug delivery system. Figure 1, details various scientific domains, which come under the field of MRI-based nanorobotics. The MRI-guided, nanorobotic system is currently a concept at its early research stages. Nevertheless, most of the modules composing this complicated system have already been developed and their functionality has reached a sufficient level of maturity. The great challenge towards the realization of the MRI-based guidance of nanoscale robotic capsules lies on the integration of the individual modules.

It is the goal of this paper to explain the importance of an MRI-based nanorobotic drug delivery system, present its main building blocks, describe the current activities in this field and discuss the challenges that have to be addressed in the near future. 


\section{Concept of Controlled Drug Delivery Systems}

The concept of controlled drug delivery systems (DDS) is to deliver drugs to the right place, at the right concentration for the right period of time. The dosage and its administration rate should result in drug temporal and spatial concentrations that are below a toxic drug level and above a level of minimal therapeutic effect. This range of desired drug concentration is called therapeutic window. Unfortunately, the body does not handle drugs in a way that maintains the drug time-profile or distribution-profile within the desired therapeutic window. On one hand, systemic circulation will distribute the drug throughout the body and consequently systemic toxicity will affect organs, which are in no-need for the drug, and on the other hand, the body always tries to remove foreign substances including drugs according to a clearance rate. It is evident that to attain the idealized time profile, a drug should be administered in a controlled way. Two types of control over drug release can be achieved: temporal and distribution control (13).

For temporal control of drug concentration, a controlled release mechanism is used, which ensures that the rate of drug release matches the rate of drug elimination. Consequently, the drug concentration remains within the therapeutic window for the vast majority of the $24 \mathrm{~h}$ period (13). Controlled release mechanisms are usually based on polymer capsules that either delay the dissolution of drug molecules, or inhibit the diffusion of the drug out of the device, or control the flow of drug solutions (13).

For distribution control, the drug is targeted and released to the precise site of activity within the body. The benefit of this type of control is shown schematically in Figure 2, in which drug concentration at the lesion and concentration systemically distributed are compared. Evidently, distribution control techniques allow to significantly increase the drug dosage while avoiding the side effects on healthy tissues. As a result the therapeutic efficiency of the treatment is drastically improved and the intensity of the side effects is minimised.

Distribution control of drug concentration is usually achieved by targeting mechanisms. A vast and diverse range of targeting mechanisms has been proposed in the literature $(40,41)$. The MRI-guided drug delivery is an active targeting mechanism that provides simultaneous propulsion and imaging capabilities, 
which allow the implementation of real-time feedback control of the targeting process. Consequently using MRI-guided drug delivery results in a high accuracy distribution control of the drug at the lesion.

\section{Overview of MRI-DDS System Architecture}

The generic architecture of the MRI-based nanorobotic system is depicted in Figure 3. The most critical components of the architecture of MRI-guided nanorobotic system are described next.

\subsection{Nanocapsule}

This is the most important subsystem of the MRI-based nanorobotic architecture. Its components are:

- The nanocarrier: This constitutes the main body of the nanocapsule and serves (i) as the vesicle for loading drug molecules and (ii) as a structure, onto which biomolecules and polymer chains are attached to provide solubility, biocompatibility and sustained circulation.

- Magnetic nanoparticles as actuating elements: These are the mechanisms responsible for the generation of propulsion forces and torques on the nanocapsule. Actuating elements are usually magnetic nanoparticles that have been encapsulated or attached on the carrier, which by exploiting the MRI field gradients induce magnetic forces and torques and serve as the propulsion mechanism of the nanocapsule.

- Surface functionalization as sensing element: These are mechanisms that sense the status of the environment surrounding the nanocapsule. Such sensing elements - usually based on biomolecules - serve various purposes, like detecting and targeting specific molecules, triggering drug release mechanisms and avoiding immunogenic reactions.

- Drug molecules: In therapeutic applications, the nanocapsules are loaded with drug molecules. Depending on the chemical process used for loading, the drug may be encapsulated in the hollow space of a vesicle or chemically attached on the surface of the carrier.

\subsection{MRI Propulsion Unit}

Every MRI installation will at least contain the following main hardware building blocks. Their functionality is shared by the propulsion system and the tracking system. 
- Main magnet: The static magnetic field is the most important and most expensive component of a MRI system. A field strength of $0.5 \mathrm{~T}$ can be achieved using permanent magnets. For higher field strengths super-conducting electromagnets are needed. Typically, liquid helium is used as the coolant to preserve the super-conducting properties.

- Gradient coils: Three gradient coil system arrangements are needed in an MRI system. The gradient coils are used for both propulsion and tracking. In the case of propulsion the gradient coils generate gradient fields, which in combination with the magnetic properties of the nanoparticles attached on or encapsulated in the nanocarrier, induce the actuation forces and torques that drive the nanocapsule.

- $\quad$ RF coils: These coils serve as a receiver and transmitter for radio frequency (RF) signals, which can be excitation of refocusing pulses as well as response signals, resulting from the nuclear magnetic resonance. Beside the built-in RF coil system of a typical MRI installation, hundreds of installation-specific coils are available for special examinations (hand, knee, etc).

- Computer system: A distributed computer system is needed to control all components of the MRI system. It will at least carry out image reconstruction and control of the gradient fields and RF coils.

\subsection{MRI Tracking Unit}

In the case of tracking, the gradient coils - described previously - are used for spatial encoding of the MR signals and echo formation. These signals are then processed by a tracking software module that comprises the (a) MRI image reconstruction software and (b) the image processing software. Its role is to estimate the position and accumulation of the nanocapsules within the vasculature, the tissues and the organs of the human. The image processing software has to run as near as possible to the image generation, to avoid communication latencies and delays (over several hundred $10^{-3} \mathrm{se}$ ) by the transfer of images which can hinder fast controller reaction.

\subsection{Controller}

- Real time control algorithm: High-level controller i.e. endovascular navigation can be accomplished by 
integrating real-time control algorithms with MRI propulsion system and tracking events. The control navigation algorithm is coordinated through the development of proprietary control modules embedded in the clinical MRI system. Optimal navigation performance requires different trade-offs in terms of refresh rate, duty cycle of the propulsion gradients, repetition time of the tracking sequence. Automatic and stable trajectory tracking require robust controller implementation by "plug-in" control architectures without modifying the hardware of clinical MRI systems. Different perturbations should be taken into account during the controller design process.

- Graphical User Interface: This module interfaces the MRI machine to the Human Operator. It comprises input command prompt, 3D-visualization and process supervision tools.

The overall concept of the in-vivo MRI-guidance system is based on the fact that both imaging and propulsion is possible by employing the gradient coils of the MRI system. Software-based upgrading of a clinical MRI system is the least expensive approach to convert a platform that is used for imaging to an effective interventional platform. At any instant only one of the functions can be applied, i.e. either imaging or guidance. However, both can be executed over the same MRI interface. For the implementation of a system capable of imaging and of propelling these particles, the MRI interface has to be shared, and a timedivision-multiple-access scheme for it has to be developed.

In vivo-demonstration of a complete MRI guided nanorobotic system has not been done so far. However, there is substantial work that has been performed to demonstrate feasibility in several of its component modules. In the sections below we describe the state of the art in several important modules for the development of MRI guided nanorobotic systems.

\section{Nanocarrier Technologies}

The potential applications of nanoparticles as therapeutic and diagnostic agents in medicine are vast. Nanoparticle-based therapeutics have enormous potential in addressing the failures of traditional therapeutics due to such factors as poor solubility or lack of target specificity. Although there are only a few Food 
and Drug Administration (FDA) approved nanoparticle based therapeutics on the market, these formulations are already impacting medicine and promise to alter healthcare (5).

Optimized nanoparticles have been developed that improve pharmacokinetics (i.e. the absorption, distribution, metabolism and excretion of the nanoparticle in the organism), and achieve more effective therapeutic activity, and minimize intensity of side effects. Critical properties of nanocarriers are biocompatibility, solubility, stability, metabolic stability, and cellular permeability. Some nanoparticles exhibit also unique optical properties, which make them not only suitable for therapeutic use but also for bio-imaging applications. The following paragraphs provide a short description of different nanoparticle technologies that could be used as the carrier module in MRI guided nanorobotic systems.

\subsection{Liposome Nanoparticles}

Liposomes are one of the most well-known drug delivery carriers employed in the treatment of cancer. Due to their advantages, liposomal formulations provide a substantial increase in antitumor efficacy comparing with the free drug or standard chemotherapy regiments. Currently, they are used as drug carriers for administration of several classes of drugs like antiviral, antifungal, antimicrobial, anti-tubercular, vaccines and gene therapeutics (42), (9). Liposomes are lipid-based vesicles of nanometer size (typically less than $400 \mathrm{~nm}$ ). Their bilayer membrane is made of two layers of phospholipids, which are molecules that have hydrophilic head (attract water) and hydrophobic hydrocarbon tail (repel water), orderly arranged as shown in Figure 4. These spherical vesicles contain a core of aqueous drug and offer effective protection of the physically entrapped solution against hydrolysis and enzymatic degradation (9).

\subsection{Polymer Micelles}

Polymers micelles have attracted significant attention as nanoscale carriers for the delivery of low mass drugs, proteins, genes, and imaging agents (43). Polymer micelles are formed by self-assembly of amphiphilic block or graft copolymers in aqueous solutions. They are characterized by a unique core-shell architecture. The core of the micelles formulates a hollow space that encapsulates water insoluble drugs or 
diagnostic agents and offers effective protection against hydrolysis and enzymatic degradation (43), (44), allowing for prolonged blood circulation times. The resulting hydrophilic shell is a brush-like corona (see Figure 5) that stabilizes the micelles in aqueous dispersion.

\subsection{Quantum Dots}

Quantum dots (QDs) are novel semiconductor nanocrystals with broad potential for use in various applications in the research, management, and treatment of cancer (45), (46), (7). They form a new class of fluorescent probes for biomolecular and cellular imaging. Also results in drug delivery have been reported in (47), where the original CdSe QD were modified by the addition of an impermeable coating of polymer that prevented the leaking of highly toxic cadmium ions from the QD conjugate and provided means to chemically attach tumor-targeting molecules $(7,48,49)$. It is known that uncoated or non-polymer protected quantum dots are unstable when exposed to UV radiation and have shown to release toxic cadmium $(48,50)$. Research has been conducted on the subject of QDs toxicity and has indicated that QDs with the appropriate stable polymer coating are essentially non-toxic to cells and animals (i.e. no effect on cell division or ATP production).

\subsection{Metallic Nanoshells}

Metallic nanoshells are colloid nanoparticles coated with a thin metallic layer to form core shell nanoparticles. Two of the most commonly used metals for the synthesis of metallic nanoshells are gold and silver. The core of the nanoshell is composed by polymers such as Teflon or Latex nanosize spheres. Other cores such as alumina, silica or titanium dioxide can also be employed. Nanoshell optical properties rely on the plasmon-mediated conversion of electrical energy into light (51). Metallic Nanoshells are most commonly used in medical applications for gene diagnostics, bioimaging, cancer imaging and intracellular analysis (52), (53). Results on drug and gene delivery using metallic nanoshells have also been reported, by Halas and co-workers in (54). Figure 6, demonstrates SEM and TEM images of silver nanoshells. 


\subsection{Gold Nanoparticles}

Gold nanoparticles also known as colloidal gold or nanogold have been used in biological applications since 1971 (49). The multifaceted abilities of gold have been investigated in depth right from the electronics industry to medicinal formulations of both modern and traditional practices. Gold nanoparticles usually have size in the range of 10-40 nm, are strong absorbers, photostable, non-toxic, easily conjugated to antibodies or proteins and have adjustable optical properties (55) (3) (56). Their optical properties are based on the surface plasmon resonance. It has been shown that nanogold has 600 times more absorption in cancer cells than normal cells. Gold nanoparticles have also been used for controlled drug delivery, where they carry drug molecules, which are released when the nanoparticles are exposed to a particular wavelength of infrared light (57).

\subsection{Carbon Nanotubes}

Carbon Nanotubes (CNTs) could also be used as nanocarriers because they have the desired dimensions, and they possess outstanding mechanical, thermal and electrical properties (58). These hexagonal networks of carbon atoms exhibit large aspect ratio (10-1000), terapascale-scale young's modulus (59), excellent elasticity (60), ultra small interlayer friction (61), and excellent field-emission properties (62). They can be fabricated in single, double or multiwall versions, open or close ended, they can be bonded together to formulate more complex structures with increased functionality and extended mechanical properties $(63,64)$. Also, it has been shown that CNTs can be filled with magnetic nanoparticles (65) (66), but, so far no high yield fabrication technique has been reported. The major limitation is the small inner diameter of CNTs and the weak nanocapilary phenomenon. Although no definite conclusions have been drawn on their biocompatibillity, on-going research shows that no apparent toxicity or negative health effects are correlated to CNTs (67).

\section{Nanoparticle functionalization for sensing purposes}

Several of the aforementioned nanoparticle properties are enhanced by appropriate nanoparticle function- 
alization, i.e. to have the nanoparticles's surface chemically modified in order to acquire desired properties. Robotic nanocapsules, are chemically processed not only for enhancing stability, solubility and biocompatibility properties but also for acquiring capabilities to sense the status of the environment surrounding the nanocapsule. Such functionalizations techniques include:

\subsection{Targeting mechanism: Functionalization with biomolecules that act as "targeting mechanisms".}

Targeting a particular type of cells is accomplished by attachment of specific molecules on the carrier's surface, thereby enhancing the binding and interactions with antigens or receptors expressed on the specific target cell populations. Typical vector molecules capable of recognizing tumors include antibodies, lectins, peptides, hormones, folate and vitamins $(68,69)$. For example, monoclonal nuclear antibodies can be attached to the drug carriers to promote drug release only to tumors cells and not to normal cells (70). Akerman et al. used quantum dots (see section 4.3) conjugated to peptides that were specific for either blood or lymphatic vessels to demonstrate specific targeting of vessels (68).

\subsection{Mechanisms for evading immunogenic reactions. Functionalization of nanocarriers with copolymers for enhanced biodistribution.}

Evading immunogenic reactions (i.e. bio-recognition and rejection of the nanocarrier by the immune system) is a critical factor for reducing clearance rates and achieving prolonged drug circulation time (for acquiring an insight on the immune system refer to reference (71)). The most well known surface modifier for avoiding the immune system is the poly(ethylene glycol) (PEG) (8).

\subsection{Triggered-release mechanisms: Functionalization of nanocarriers with copolymers containing stim- uli- responsive functionalities.}

This is an important functionalization property for the case of drug delivery systems. The use of triggeredrelease mechanism allows the nanocapsule to become an active participant, where the release of the drug can be activated according to a controlled stimulus as opposed to the conventional drug delivery systems, where the release of the drug is predetermined by the encapsulation material. Environmental changes that 
can trigger the release of drug molecules are: $\mathrm{pH}$-variations, temperature variations, optical or acoustic stimuli (72).

The idea of a multi-functionalized drug loaded nanocarrier is schematically depicted on Figure 7. It is recognized that the preparation of multifunctional magnetopolymeric nanohybrids constitutes a major challenge and is a subject of on-going research (73). The biocompatibility and system stability issues become even more crucial when the nanocarrier is drug loaded and is further modified by antibodies for enhanced targeting capabilities. Another key issue is the development of high quality, high yield fabrication techniques for multifunctionalized drug loaded nanocapsules.

\section{Magnetic Nanoparticles as Actuating Element}

Magnetic nanoparticles form the means of actuation in MRI-based nanorobotic systems. They have been used either as nanocarriers decorated with co-polymer chains or as distributed nanoparticles attached onto the surface of other nanocarriers (carbon nanotubes, polymer micelles, etc). Magnetic nanoparticles have been employed in a broad range of biomedical applications (Figure 8). For diagnostic purposes they have been tested clinically in humans for characterizing lymph node status in patients with breast cancer (74), lung cancer, prostate cancer (75) endometrial cancer and cervical cancer (76). Recently, scientists started to employ magnetic nanoparticles conjugated with drug molecules and biomolecules for drug delivery purposes, (16), (14), (77-79).

Magnetic nanocapsules are guided - by magnetic forces and torques - within the vasculature and concentrated at a localized target on a tissue. The magnitude and direction of magnetic forces and torques depend (a) on the external magnetic field and magnetic field gradients generated by the MRI coils, (b) on the magnetization properties of the magnetic nanoparticles, and (c) on the volume of the nanoparticle. These physical relations are expressed through the following equations:

$$
\begin{gathered}
\vec{F}_{m}=V_{m}(\vec{M} \nabla) \cdot \vec{B} \\
\vec{\tau}_{m}=V_{m} \vec{M} \times \vec{B}
\end{gathered}
$$

Where $\vec{F}_{m}$ is the magnetic force $(\mathrm{N}), \vec{\tau}$ is the magnetic torque $(\mathrm{Nm}), \vec{M}(\mathrm{~A} / \mathrm{m})$ is the magnetization per 
unit volume of the magnetic nanoparticle, $V_{m}\left(\mathrm{~m}^{3}\right)$ is the magnetic volume of the nanoparticle and $\vec{B}(\mathrm{~T})$ is the magnetic field.

When ferromagnetic materials scale down to approximately less than $\sim 50 \mathrm{~nm}$ their magnetic properties, at room temperature, become superparamagnetic. Superparamagnetic particles behave differently than the original ferromagnetic matter: They exhibit strong magnetization within a magnetic field, but contrary to ferromagnetic materials their magnetization $\vec{m}$ is diminished when the external magnetic field $\vec{H}$ becomes zero. The zero remanent magnetization stems from the fact that in the absence of external field $\vec{H}$, thermal agitation (even at room temperature) randomly orients magnetic dipoles resulting in zero net magnetization of the nanoparticle. Furthermore, they do not exhibit hysteresis.

The relation between $\vec{m}$ and $\vec{B}$ for saturated superparamagnetic nanoparticles is given by the following formula:

$$
\vec{m}=\frac{V M_{s} \vec{B}}{\|\vec{B}\|} L\left(\frac{V M_{s}\|\vec{B}\|}{k T}\right)
$$

where $M_{s}$ is the magnetic saturation per unit volume, $k T$ is thermal energy due to thermal agitation of the atoms and molecules. The Langevin function $L(x)$ is expressed by:

$$
L(x)=\operatorname{coth}(x)-\frac{1}{x}
$$

Simple calculations demonstrate that a field $\vec{B}$ of $30-40 \mathrm{mT}$ is sufficient to drive the magnetization of the Fe superparamagnetic nanoparticle into saturation.

The superparamagnetic properties largely depend on the actual size of the nanoparticle. Magnetic coercivity varies as a function of size as shown in Figure 9, (80). In large nanoparticles energetic considerations favor the formation of domain walls. However, when the particle size decreases below a certain value, the formation of domain walls becomes unfavorable and each particle comprises a single domain. This is the case for superparamagnetic nanoparticles (14). The transition from multi-domain material to single domain is depicted schematically in Figure 9. 
Saturation magnetization $m_{s}$ also varies as size scales down. This behavior is owed to magnetic disordered layers near the surface, i.e. surface canting effects, which become dominant when the ratio of surface over volume is substantially increased. The relation describing magnetic saturation $m_{s}$ as a function of nanoparticle size is given by:

$$
m_{s}=M_{s}[(r-d) / r]^{3}
$$

where $r$ is the size, $M_{s}$ is the saturation magnetization of bulk materials and $d$ the thickness of disordered surface layer.

A characteristic example of scaling magnetic properties is $\gamma-\mathrm{Fe}_{2} \mathrm{O}_{3}$, where nanoparticles of $55 \mathrm{~nm}$ exhibit ferromagnetic properties with a coercivity of $4138 \mathrm{~A} / \mathrm{m}$ at $300 \mathrm{~K}$, but at a size down to $12 \mathrm{~nm}$ $\gamma-\mathrm{Fe}_{2} \mathrm{O}_{3}$ nanoparticles exhibit superparamagnetic properties (80). Figure 10 demonstrates TEM images and corresponding magnetization loops of iron oxide nanoparticles, whose size is at $55 \mathrm{~nm}$ (Figure 10a,b) and at $12 \mathrm{~nm}(\mathrm{c}, \mathrm{d})$. As can be observed in plot d, superparamagnetic behaviour is characterized by (i) no hysteresis, (ii) zero coercive field and (iii) zero remanent magnetization.

Furthermore, it is known that shape characteristics like aspect-ratio also influence magnetic properties. The interested reader is referred to (80), (81) for a detailed study.

\section{MRI Based Propulsion, Tracking and Control}

Once a nanocarrier with magnetic nanoparticles has been placed inside a MRI scanner, it is important to demonstrate its controllable propulsion, tracking and navigation. This section presents the latest developments in this area of study.

\subsection{Magnetic Propulsion and Steering}

In (82) a magnetic navigation system produced by Stereotaxis, Inc. was used for the propulsion of a millimeter size endoscopic robotic capsule. Magnetic propulsion and steering for microparticles has also been employed in $(23,25)$ where the magnetic torque and force of a microrobot were induced independently by Maxwell and Helmholtz coil fields respectively. Other concepts of magnetic propulsion have also 
been proposed. One is referred to as biomimetic robots using flagella: a red blood cell is fixed to a beating flagella (33) imitating the motion of eukaryotic bacteria, or a magnetic bead is attached to a helical nanocoil $(34,35)$, like prokaryotic bacteria. Another concept is the magnetotactic bacteria. Such bacteria are actuated thanks to embedded or attached ferromagnetic material (83).

The use of magnetic gradients provided by a clinical MRI scanner to pull microparticles has been demonstrated in $(28,29)$. This approach has been demonstrated at the millimeter and micrometer scales where low level multiplexed controllers and observers have been developed (84), and in-vivo experiments have been performed on a living animal (85) (though the blood flow had been stopped using a balloon catheter). However, great technical challenges are encountered as the particle's size reduces down to the nanoscale. Magnetic forces used for propelling are volumetric, whereas the drag force is --at best-- dependant on the particles' area, therefore the smaller the particle, the higher the magnetic gradients should be for efficiently propelling the particles against the drag force. Consequently, the MRI propulsion is well conditioned for particles whose radius is up to a few dozen micrometers with current MRI scanners. At lower scales, the required gradients are well above those generated by clinical MRIs. Currently, research groups investigate approaches for increasing the magnetic forces acting on the particles. One of them is the use of additional coils to supply higher gradients (24). MRI systems upgraded with additional gradient steering coils in order to increase standard MRI gradient amplitudes $(100-500 \mathrm{mT})$ is currently investigated (80). Another promising approach is to exploit the aggregation properties of the magnetic micro/nano particles (see Figure 11). The formation of aggregations, due to the MRI field, increases the magnetic volume, which in turn gives rise to larger ratio of magnetic forces over drag forces (86-88). An alternative approach is followed in (89), where drug molecules and magnetic nanoparticles are encapsulated into larger biodegradable polymer microcarriers. In this work the MRI scanner, using additional gradient coils, steers the polymer microcarriers in the blood vessels near the tumor and embolizes the surrounding capillaries. The embolized polymer carriers biodegrade and release the encapsulated therapeutic agents, which are then directed into the tumor. A combination of all three aforementioned approaches is expected to yield the maximum targeting efficiency. 


\subsection{Imaging and Tracking in MRI Scanners}

Hydrogen atoms, as the main component of water, are widely spread in the human body, and various tissues can be identified with respect to the hydrogen's density and stability. MRI imaging is based on exciting these atoms and let them relax to equilibrium to obtain a map of their repartition and properties, and thus to get an image of the body tissues in the observed slice. More precisely, clinical MRI imaging can be broken into four major steps. First, the permanent magnet generates a strong and uniform magnetic field in order to align the spin magnetic moment of hydrogen nuclei (protons) along this field. Once polarized, protons are excited by a pulsing RF coil at Larmor frequency to induce a resonance absorption, which modifies the precession motion of the spin. Third, RF excitation is stopped and protons relax to their equilibrium state, producing a free induction decay signal (FID) in the RF coil after some relaxing times. Meanwhile, gradient coils are used first to select the slice to be observed during the polarization step, secondly to perform a phase shift/ frequency-encoding during the readout step, performing a 2D position encoding within the slice. Finally, the slice image is obtained thanks to inverse Fourier transforms applied on the measured FID. Precision of an MRI imaging system is strongly related to the uniformity and strength of the main magnet on the one hand, since they decrease the signal to noise ratio. On the other hand, speed and strength of the gradient coils (set by their duty cycle) also affect precision and scanning speed of the imager.

The use of MRI as imaging modality for 3D image-guidance of endovascular procedures is of great interest. As already mentioned endovascular navigation is rendered possible by integrating propulsion and tracking events using time multiplexing. The same equipment that induces motion to nanororobots can be utilized for localization by combining imaging/tracking procedures for precise navigation of an untethered interventional nanorobot. The main drawback of MRI localization is that the choice of material for fabrication of the microrobot is limited. Ferromagnetic objects cause image artifacts that are sometimes larger than the object itself to be localized, even though information contained in spatial gradients can overcome this limitation (90). Efforts to increase MRI sensitivity have focused on the development of new magnetic core materials (91), or on the improvement in nanoparticle size (92) or clustering (93). An emerging theme in 
nanoparticle research is to control biological behavior and/or electromagnetic properties by controlling shape. Swarms of nanoparticles were localized in an MRI using contrast agents (94), and swarms of magnetotatic bacteria as a single object in (37). In (95), the authors found that a nanostructure with an elongated assembly of nanoparticles (referred to as nanoworms) influences their efficacy both in vitro and in vivo by enhancing their magnetic relaxivity in MRI. In addition to the trade-off between refresh rate and duty cycle of the propulsion gradients, the repetition time of the tracking sequence is another constraint to be taken into account during imaging and tracking sequences (84). With insufficient repetition time, MRI signal can be unusable for tracking. Fast and robust image processing algorithms are key issues for future research.

\subsection{Multiscale Modeling and Computational Tools}

Magnetic drug delivery is a multiphysics process involving diverse physical domains such as magnetic fields and fluid dynamics. It is also a multiscale process ranging from the mini dimensions of the arteries to the micro dimensions of the capillaries and the microparticles, and down to the nano dimensions of the single domain superparamagnetic particles. Evidently there is not a complete modeling framework and there is no single software package for simulating the entire process, since this would require the integration of multiparticle simulation, molecular simulation, continuum-based models, stochastic methods and nanomechanics. A number of deterministic dynamic modeling approaches for magnetic targeting are presented in $(23,78,80,87,88,96-98)$. Nonetheless, critical physical parameters of endovascular navigation are captured by the simplified model depicted in Figure 13, where the untethered nanocapsule is subjected to the apparent weight, magnetic force and torque, and hydrodynamics.

The apparent weight is the combined action of weight and buoyancy:

$$
\vec{W}_{a}=V\left(\rho_{b}-\rho_{f}\right) \vec{g}
$$

As it was mentioned in the previous section the magnetic force is related to the magnetic field gradients:

$$
\vec{F}_{m}=V_{m}(\vec{M} \nabla) \cdot \vec{B}
$$

Under the assumption of a Newtonian incompressible blood flow and that the Reynolds number is low, the fluidic drag force can be simplified according to the Stokes formula: 


$$
\vec{F}_{d}=6 \pi \eta r \vec{U}
$$

with $\eta$ the dynamic viscosity of blood, $\mathrm{r}$ the Stokes radius and $\vec{U}$ the relative velocity of the particle with respect to the flow.

The non-dimensional number $C M_{D}$ given by Eq (9) is characteristic of a magnetic targeting system. The design of the nano/micro capsule should pursuit maximization of $C_{M D}$. The lower the value of $C_{M D}$ (for MRI DDS usually is $\left.C_{M D}<1\right)$ the more difficult it is to navigate the capsules.

$$
C_{M D}=\frac{F_{m a g}}{F_{d}}=\frac{V\left(M_{s} \cdot \nabla\right) B}{6 \pi \eta r U}>1
$$

The lack of strong computational tools for the range of interest is a key issue for future research. Molecular and atomic scales can be simulated using Molecular Dynamics tools, while sizes greater than 500 $\mathrm{nm}$ are modeled either using discrete element methods or using CFD tools. But still there are no systematic simulation environments for studying the dynamics of the ferrofluid, as well as for conceptualization, characterization, development and prototyping of nanocapsules. A macroscale approach has been followed in (99), where a FEM package has been used for 3D computational simulations of blood flow and magnetic particle motion in a left coronary artery and a carotid artery. The critical effect of aggregation and also the forces of electrostatic nature have been neglected. A different approach has been adopted in $(87,88)$, where a 2D simulation platform for magnetic targeting of microparticles has been designed and developed. The simulation platform is based on discrete element modeling techniques and predicts the size and geometry of the aggregations (Figure 14), and their response during magnetic propulsion. Its modular design allows the incorporation of particle geometries other than sphere and also the use of a feedback control loop. No turbulent blood flow is taken into account.

\subsection{Control Algorithms for Navigation in MRI Environment}

Endovascular navigation through MRI system will be feasible by integrating propulsion and tracking events within the control software that deals with their time sequencing. The MRI interface has therefore to be shared and a time-division-multiple-access scheme for it has to be developed. Optimal navigation performance will require different tradeoffs in terms of refresh rate, duty cycle of the propulsion gradients, repeti- 
tion time of the tracking sequence. Recently, the authors in $(29,38,85)$, demonstrated that a ferromagnetic particle was navigated in real-time along pre-planned trajectories in the carotid artery of living swine at an average velocity of $10 \mathrm{~cm} . \mathrm{s}-1$. As the propulsion module used a simple proportional-derivative-integral (PID) controller (84), the authors reported that navigations instabilities occurred during experiments. At the nanoscale, automatic and stable trajectory control of nanocapsules against environment perturbations (blood flow, drag wall forces) will require advanced controller implementation without modifying the hardware of clinical MRI system for two main reasons:

Magnetic gradients are used both for observation and control purposes in a time-multiplexed sequence. Although the uniform fields of commercial MRI machines are quite high $(1.5-3.0 \mathrm{~T})$, the gradient fields they can generate continuously are in the -0.08 to $0.08 \mathrm{~T} / \mathrm{m}$ range and are limited by the capacity of the non super-conducting gradient coils needed for the rapidly changing field. The time-multiplexed sequence design, combined with the MRI overheating avoidance leading to limitations on the MRI duty cycle, tends to increase the disproportional scaling between magnetic forces used for control purpose and perturbation forces (drag forces and net buoyancy forces).

Magnetic forces cannot generically propel the nanocapsule against the blood flow. Therefore, control objectives may be relaxed into steering the nanocapsule within the vessels bifurcations. Predictive controllers including the capsule's motion and dynamics with estimation of the versatile flow due to heart pumping are of great interest.

To this end, a system software architecture, has been proposed and is shown in Figure 15 illustrating the different software modules to allow 3D navigation of a microdevice in blood vessels (100), namely: (i) vessel path extraction, (ii) magnetic gradient steering, (iii) tracking and (iv) closed-loop navigation control. First, the navigation path of the microrobot into the blood vessel is extracted using Fast Marching Method from the pre-operation images (3D MRI imaging) to guide the microrobot from the injection point to the tumor area through the anarchic vessel network. Then, this extracted path can be optimized so as to minimize the control efforts (i.e. the magnetic gradients amplitude) required to propel the particle, which 
lead to an optimal reference trajectory (101).

Based on this optimal trajectory, a Model Predictive Controller (MPC) is proposed for robust timemultiplexed navigation along a 3D path in presence of pulsative flow. Due to nonlinearities of the particle's dynamics, Lyapunov based controllers have to be considered to increase navigation performances. Such observer-controller pairs have lately been proposed (102), (103) and proved more robust than PID approaches on simulation results, with respect to measurement noise or to some matched uncertainties (101). Let us notice that the low-level controller also provides robustness performances against MRI system constraints (as pulsatile flow, limited gradient amplitude, and image noise) while the Model Predictive Controller (MPC) is designed to trade-off time-multiplexed sequence constraints in terms of refresh-rate, duty cycle of the propulsion gradients, and repetition time of the tracking sequence.

\section{Discussion}

MRI DDS is a promising approach for targeting therapeutic agents through intravascular roots. A significant advantage of the MRI DDS is that the three linearly independent, time-varying, magnetic gradients can target deep-seated lesions in the human body. This requirement cannot be met by the use of static permanent magnets. The use of MRI significantly increases the targeting accuracy and the capturing efficiency of the drugs due to the trajectory tracking capability provided by its imaging modality. Finally, MRI installations exist in many hospitals around the world and therefore this approach does not incur excessive cost.

Research on the field of DDS using clinical MRI is facing significant challenges especially when it comes to the efficient real-time propulsion and position detection of the nano or micro particles. The greatest challenge so far is the disproportional scaling of magnetic forces over drag forces. In the nano dimensions these scaling effects in combination with the low magnetic gradients generated by the MRI result in poor targeting efficiency of the MRI DDS. The magnetic gradients are low because they are limited by the maximum permissible current flowing in the gradient coils. Also, there are biological limits that prohibit applying magnetic gradients of high magnitude and high frequency on human subjects. Ongoing research aims to overcome the aforementioned constraints by: 
(i) Enhancing the gradient coils of the MRI without incurring significant cost $(27,29,38)$.

(ii) Augmenting the magnetic volume of the guided therapeutic agents either by performing aggregation techniques (86-88) or by encapsulating the therapeutic agents inside biodegradable polymers (89). The efficiency of the former approach is limited by the dispersion of the navigated aggregations along the vessel axis due to turbulent effects and also by the algorithmic complexity of the swarmtype navigation needed to manipulate the aggregated objects. The polymer capsule approach has been successfully tested in a phantom mimicking rabbit liver artery, but it has not been applied yet at a human scale.

(iii) Exploiting novel magnetic materials whose magnetic properties are superior to those of iron-oxide superparamagnetic nanoparticles (104). The hurdle in this approach is testing and ensuring the biocompatibility of these material as well as developing high yield fabrication techniques.

Further challenges are posed by the imaging system. Specifically the MRI, cannot yet provide high frame rate feedback with sufficient resolution to reliably control the robot. Frame rate requirements become even tougher when considering that blood flow becomes turbulent in select areas of the body. Fast acquisition and processing is necessary to later enable real-time control of the nanocapsule. The time consumed for imaging purposes should be reduced as much as possible, to enable stronger and longer propulsion gradients. Considerable research effort is put on the development of imaging processing software that has to run as near as possible to the image generation, to avoid communication latencies and delays by the transfer of images which can hinder fast controller reactions.

A key issue is the functionalization of drug-loaded nanocarriers, with biomolecules, copolymers and superparamagnetic nanoparticles, while satisfying requirements of stability, biodistribution and biocompatibility. Fabrication issues have to be addressed as well. For example carbon nanotubes are very promising as nanocapsules, but their use is limited by the lack of systematic fabrication of high quality, high yield of magnetic nanotube capsules for drug containment, furthermore there is a growing debate over the toxic effects of carbon nanotubes and their potential use for biomedical applications such as drug delivery vehi- 
cles.

It is recognized that due to the system stringent requirements and due to the aforementioned technical limitations, the development of the fully functional MRI DDS is especially challenging. However, technological and scientific advancement on the fields of MRI imaging, of nanocapsule fabrication and of nanorobotic manipulation is fast and it is expected that soon the most critical components of the system will reach a technological maturity sufficient to provide the first fully functional MRI DDS.

\section{Conclusions}

This paper reviewed the novel concept of guiding nanorobotic capsules in the human vasculature for drug delivery purposes using a MRI scanner. The successful realization of the MRI DDS is expected to result in significant increase of the therapeutic efficiency and in reduction of the side effects on healthy tissue. The system architecture of the MRI DDS comprises functionalized drug-loaded nanocapsules, MRI propulsion module, MRI tracking module, a controller and a User Interface. A fully functional MRI DDS has not been developed yet, and it is recognized that its development is especially challenging due to the extreme scaling effects and due to the integration of diverse technologies subject to very stringent requirements. On the other hand owing to research breakthroughs on the fields of nanoparticle manipulation, of MRI imaging, of nanocapsule fabrication and magnetic materials, the critical components of the system architecture are reaching a sufficient level of technological maturity and this nanorobotic concept is now on the verge of realization.

\section{Acknowledgments}

This work was supported by European Union's 7th FWP (Seventh Framework Programme) and its research area: ICT-2007.3.6 Micro/nanosystems under the project NANOMA: Nano-Actuators and Nano Sensors for Medical Applications. 


\section{References}

1. Ummat A, Dubey A, Mavroidis C. 2006. Bio-nanorobotics: A Field Inspired by Nature. In Biomimetics: Biologically Inspired Technologies, ed. Y Bar-Cohen. Boca Raton London New York: CRC Taylor \& Francis

2. Berry CC, Curtis ASG. 2003. Functionalization of magnetic nanoparticles for applications in biomedicine. Journal of Physics D: Apllied Physics 36: 198-206

3. Hede S, Huilgol N. 2006. Nano: The new nemesis of cancer. J Cancer Res Ther 2: 186-95

4. Gupta RB, Kompella UB. 2006. Nanoparticle Technology for Drug Delivery. New York Taylor \& Francis

5. Bawa R. 2008. Nanoparticle-based Therapeutics in Humans: A Survey. Nanotechnology Law \& Business 5: 135-55

6. Bhushan B, ed. 2004. Handbook of Nanotechnology: Springer-Verlag

7. Cuenca AG, Jiang H, Hochwald SN, Delano M, Cance WG, Grobmyer SR. 2006. Emerging Implications of Nanotechnology on Cancer Diagnostics and Therapeutics. Cancer 1: 459-66

8. Kingsley JD, Dou H, Morehead J, Rabinow B, Gendelman HE, Destache CJ. 2006. Nanotechnology: A Focus on Nanoparticles as a Drug Delivery System. J Neuroimmune Pharmacol 1: $340-50$

9. Kiparissides C, Kammona O. 2008. Nanotechnology advances in controlled drug delivery systems. Physica Status Solidi (c) 5: 3828-33

10. Langer R. 2001. Perspectives: Drug delivery - drugs on target,. Science 293: 58-59

11. Orive G, Hernandez RM, Gascon AR, Pedraz JL. 2005. Micro and nano drug delivery system in cancer therapy. Cancer Therapy 3: 131-38

12. Sengupta S, Eavarone D, Capila I, Zhao G, Watson N, et al. 2005. Temporal targeting of tumour cells and neovasculature with a nanoscale delivery system. Nature Letters 436: 568-72 
13. Uhrich K, Cannizaro S, Langer R, Shakeseff K. 1999. Polymeric Systems for Controlled Drug Release. Chemical Reviews 99: 3181-98

14. Arruebo M, Fernandez-Pacheco R, Ibarra MR, Santamaria J. 2007. Review: Magnetic nanoparticles for drug delivery. NanoToday 2: 22-32

15. Dobson J. 2006. Magnetic micro- and nano-particle-based targeting for drug and gene delivery. Nanomedicine 1: 31-37

16. Misra RDK. 2008. Magnetic nanoparticle carrier for targeted drug delivery: perspective, outlook and design. Materials Science and Technology 24: 1011-19

17. Pankhurst QA, Connolly J, Jones SK, Dobson J. 2003. Review: Applications of magnetic nanoparticles in biomedicine. JOURNAL OF PHYSICS D: APPLIED PHYSICS 36: 167-81

18. Gillies GT, Ritter RC, Broaddus WC, Grady MS, Howard MA, McNeil RG. 1994. Magnetic manipulation instrumentation for medical physics research. Rev. of Sci. Instruments 65

19. Alexiou C, Jurgons R, Seliger C, Brunke O, Iro H, Odenbach S. 2007. Delivery of Superparamagnetic Nanoparticles for Local Chemotherapy after Intraarterial Infusion and Magnetic Drug Targeting. Anticancer Research 27: 2019-22

20. Grief AD, Richardson G. 2005. Mathematical modelling of magnetically targeted drug delivery. Journal of magnetism and magnetic materials 293: 455-63

21. Quate EG, Wika KG, Lawson MA, Gillies GT, Ritter RC, et al. 1991. Goniometric motion controller for the superconducting coil in amagnetic stereoaxis system. IEEE Trans. on Biomed. Eng. 38: 899-905

22. Takeda S-I, Mishima F, Fujimoto S, Izumi Y, Nishijima S. 2006. Development of magnetically targeted drug delivery system using superconducting magnet. Journal of Magnetism and Magnetic Materials 311: 367-71 
23. Abbott JJ, Ergeneman O, Kummer MP, Hirt AM, Nelson BJ. 2007. Modeling Magnetic Torque and Force for Controlled Manipulation of Soft-Magnetic Bodies. IEEE Transactions on Robotics 23: $1247-52$

24. Yesin KB, Vollmers K, Nelson BJ. 2006. Modeling and control of untethered biomicrorobots in a fluidic environment using electromagnetic fields. Int. J. Robot. Res. 25: 527-36

25. Abbott JJ, Peyer KE, Lagomarsino MC, Zhang L, Dong L, et al. 2007. How should microrobots swim? Int. Symp. of Robotics Research: In press

26. Martel S, Felfoul O, Mathieu JB, Chanu A, Tamaz S, et al. 2009. MRI-based Medical Nanorobotic Platform for the Control of Magnetic nanoparticles and Flagellated Bacteria for Target Interventions in Human Capillaries. Interantional Journal of Robotic Research In press

27. Martel S, Mathieu J-B, Felfoul O, Chanu A, Aboussouan E, et al. 2008. A computer-assisted protocol for endovascular target interventions using a clinical MRI system for controlling untethered microdevices and future nanorobots. Computed Aided Surgery 13: 340-52

28. Mathieu J, Beaudoin G, Martel S. 2006. Method of Propulsion of a Ferromagnetic Core in the Cardiovascular System Through Magnetic Gradients Generated by an MRI System. IEEE Transactions on Biomedical Engineering 53: 292-99

29. Mathieu J-B, Martel S. 2007. Magnetic microparticle steering within the constraints of an MRI system: proof of concept of a novel targeting approach. Biomedical Microdevices 9: 801-08

30. Mathieu J-B, S. Martel, Yahia LH, Soulez G, Beaudoin G. 2003. MRI Systems as a Mean of Propulsion for a Microdevice in Blood Vessels. Presented at IEEE EMBS, Cancum, Mexico

31. Rosengart AJ, Kaminski MD, Chen H, Caviness PL, Ebner AD, Ritter JA. 2005. Magnetizable implants and functionalized magnetic carriers: A novel approach for noninvasive yet targeted drug delivery. Journal of magnetism and magnetic materials 293: 633-38

32. Yellen BB, Forbes ZG, Halverson DS, Fridman G, Barbee KA, et al. 2005. Targeted drug delivery to magnetic implants. Journal of magnetism and magnetic materials 293: 647-54 
33. Dreyfus R, Beaudry J, Roper ML, Fermigier M, Stone HA, Bibette J. 2005. Microscopic artificial swimmers. Nature 437: 862-65

34. Behkam B, Sitti M. 2006. Design methodology for biomimetic propulsion of miniature swimming robots. ASME Journal of Dynamic Systems, Measurement and Control 128: 36-43

35. Behkam B, Sitti M. 2007. Bacterial flagella-based propulsion and on/off motion control of microscale objects. Applied Physics Letters 90 1-3

36. Lee H, Purdon AM, Chu V, Westervelt RM. 2004. Controlled assembly of magnetic nanoparticles from magnetotactic bacteria using microelectromagnets arrays. Nano Letters 4: 995-98

37. Martel S, Mohammadi M, Felfoul O, Lu Z, Pouponneau P. 2009. Flagellated magnetotactic bacteria as controlled MRI-trackable propulsion and steering systems for medical nanorobots operating in the human microvasculature. Int. Journal of Robotics Research 28: 571-82

38. Mathieu J-B, Martel S. 2007. In Vivo Validation of a Propulsion Method for Untethered Medical Microrobots Using a Clinical Magnetic Resonance Imaging System. In IEEE/RSJ International Conference on Intelligent Robots and Systems, pp. 502-08. San Diego California US

39. Darton NJ, Sederman AJ, Ionescu A, Ducati C, Darton RC, et al. 2008. Manipulation and tracking of superparamagnetic nanoparticles using MRI Nanotechnology 19

40. Jair Bar, Roy S Herbst, Onn A. 2009. Targeted drug delivery strategies to treat lung metastasis. Expert Opinion: Drug Delivery 6: 1003-16

41. Torchilin V. 2010. Passive and active drug targeting: drug delivery to tumors as an example. Handb Exp Farmacology 197: 3-53

42. Immordino ML, Dosio F, Cattel L. 2006. Stealth liposomes: review of the basic science, rationale, and clinical applications, existing and potential. Int J Nanomedicine 1: 297-315

43. Bontha S, Kabanov A, Brovnich T. 2006. Polymer micelles with cross-linked ionic cores for delivery of anticancer drugs. Journal of Controlled Release 114: 163-74 
44. Kwon GS, Kataoka K. 1995. Block copolymer micelles as long-circulating drug vehicles. Adv. Drug. Deliv. Rev. 16: 295-309

45. Bruchez MJ, Moronne M, Gin P, Weiss S, Alivisatos A. 1998. Semiconductor nanocrystals as fluorescent biological labels. Science 281: 2013-16

46. Seydel C. 2003. Quantum dots get wet. Science 300: 80-81

47. Gao X, Cui Y, Levnson RM, Chung LWK, Nie S. 2004. In vivo cancer targeting and imaging with semiconductor quantum dots. Nat Biotechnol. 22: 969-76

48. Derfus A, Chan W, Bjatia S. 2004. Proboing the cytotoxicity of semiconductor quantum dots. Nano Letters 4: 11-18

49. Vo-Dinh T, ed. 2007. Nanotechnology In Biology and Medicine: CRC Press

50. Hobbs S, Monsky W, Yuan F. 1998. Regulation of transport pathways in tumour vessels: role of tumour type and microenvironment. Proc Natl Acad Sci USA 95: 4607-12

51. Alper J. 2005. Shining a light on cancer research. In NCI Alliance for Nanotechnology in Cancer, Monthly Feature, pp. 1-3: National Cancer Institute

52. Anker JN, Behrend C, Kopelman R. 2003. Magnetically modulated optical nanoprobes. Appl Phys Lett 82: 1102-04

53. Vo-Dinh T, Yan F, Wabuyele MB. 2005. Surface-enhanced Raman scattering for medical diagnostics and biological imaging. J Raman Spectrosc 36: 640-47

54. Sershen SR, Westcott SL, Halas NJ, West JL. 2000. Temperature-sensitive polymer-nano-shells composites for photthermally modulated drug delivery. J Biom Mat Res 51: 293-98

55. Letfullin RR, Joenathan C, George TF, Zharov VP. 2006. Laser-induced explosion of gold nanoparticles: potential role for nanophotothermolysis of cancer. Nanomedicine 1: 473-80

56. Wostek-Wojciechowska D, Jeszka JK, Uznanski P, AMiens C, Chaudret B, Lecante P. 2004. Synthesis of gold nanoparticles in solid state by thermal decomposition of an organometallic precursor. Materials Science-Poland 22: 407-13 
57. Wijaya A, Schaffer SB, Pallares IG, Hamad-Schifferli K. 2008. Selective Release of Multiple DNA Oligonucleotides from Gold Nanorods. ACS NANO 3: 80-86

58. Bhushan B, ed. 2005. "Handbook of Nanotechnology": Springer-Verlag

59. Yu MF. 2000. Tensile Loading of Ropes of Single Wall Carbon Nanotubes and their Mechanical Properties. Phys. Rev. Lett. 84: 5552-55

60. Walters DA, Ericson LM, Casavant MJ, Liu J, Colbert DT, et al. 1999. Elastic strain of freely suspended single-wall carbon nanotube ropes. Appl Phys Lett 74: 3803-05

61. Kis A, Jensen K, Aloni S, Mickelson W, Zettl A. 2006. Interlayer Forces and Ultralow Sliding Friction in Multiwalled Carbon Nanotubes. Phys. Rev. Lett. 97: 025501-4

62. Walt AdH, Chatelain A, Ugarte D. 1995. A Carbon Nanotube Field-Emission Electron Source. Science 270: 1179-80

63. Dong LX, Shou K, Frutiger DR, Subramanian A, Zhang L, et al. 2008. Engineering Multi-walled Carbon Nanotubes Inside a Transmission Electron Microscope Using Nanorobotic Manipulation. IEEE Transactions on Nanotechnology Vol. 7: 508-17

64. Dong LX, Subramanian A, Shou KY, Nelson BJ. 2006. Engineering Multi-Walled Carbon Nanotube Shells by Electric Breakdown. Presented at European nano Systems 2006, Paris, France

65. Liu YF, Wang HF. 2007. Nanomedicine - Nanotechnology tackles tumours. Nature Nanotechnology 2: pp. 20-21

66. Mitchel DT, Lee SB, Trofin L, Li NC, Nevanen TK, et al. 2002. Smart nanotubes for bioseparations and biocatalysis. Journal of the American Chemical Society 124: 11864-65

67. Liu Z, Cai WB, He LN, Nakayama N, Chen K, et al. 2007. In vivo biodistribution and highly efficient tumour targeting of carbon nanotubes in mice. Nature Nanotechnology 2: pp. 47-52

68. Akerman M, Chan W, Laakkonen P, Bhatia S, Ruoslahti E. 2002. Nanocrystal targeting in-vivo. Proc Natl Acad Sci USA 99: 12617-21 
69. Paciotti G, Myer L, Weinreich D, al e. 2004. Colloidal gold: a novel nanoparticle vector for tumor directed drug delivery. Drug Deliv. 11: 169-83

70. Iakoubov L, Rokhlin O, Torchilin V. 1995. Antinuclear autoantibodies of the aged reactive against the surface of tumor but not normal cells. Immunol Lett 47: 147-49

71. Purves WK, Sadava D, Orians GH, Heller HC. 2008. Life - the science of biology: W. H Freeman \& Company

72. Shenoy D, Little S, Langer R, Amiji M. 2005. Poly(ethylene oxide)- modified poly( $\beta$-amino ester) nanoparticles as a $\mathrm{pH}$-sensitive system for tumor-targeted delivery of hydrophobic drugs: Part 2. In vivo distribution and tumor localization studies. Pharm Res 22: 2107-14

73. Jensen GC, Yu X, Gong JD, Munge B, Bhirde A, et al. 2009. Characterization of MultienzymeAntibody-Carbon Nanotube Bioconjugates for Immunosensors. Journal of Nanoscience and Nanotechnology 9: 249-55

74. Michel S, Keller T, Fronhlich J. 2002. Preoperative breast cancer staging: MR imaging of the axilla with ultrasmall superparamagnetic iron oxide enhancement. Radiology 225: 527-36

75. Nguyen BC, Stanford W, Thompson BH. 1999. Multicenter clinical trial of ultrasmall superparamagnetic iron oxide in the evaluation of mediastinal lymph nodes in patients with primary lung carcinoma. J. Magn Reson Imagning 10: 468-73

76. Rockall A, Sohaib S, Harisinghani M. 2005. Diagnostic performance of nanoparticle-enhanced magnetic resonance imaging in the diagnosis of lymph node metastases in patients with endometrial and cervical cancer. J Clin Oncol 23: 2813-21

77. Balakrishnan S, Bonder MJ, Hatjipanayis GC. 2009. Particle size effect on phase and magnetic properties of polymer-coated magnetic nanoparticles. Journal of magnetism and magnetic materials 321: $117-22$

78. Cregg P, Murphy K, Mardinoglu A. 2008. Calculations of nanoparticle capture efficiency in magnetic drug targeting. Journal of Magnetism and Magnetic Materials 320: 3272-75 
79. Jurgons R, Seliger C, Trahms AHL, Odenbach S, Alexiou C. 2006. Drug loaded magnetic nanoparticles for cancer therapy. Journal of Physics: Condenced Matter 18: 2893-902

80. Jun Y-W, Seo J-W, Cheon J. 2007. Nanoscaling laws of magnetic nanoparticles and their applicabilities in biomedical sciences. Accounts of chemical research 41: 179-89

81. Jales D. 1998. Introduction to Magnetism and Magnetic Materials. Boca Raton FL: CRC Press

82. Carpi F, Pappone C. 2008. Magnetic Manouvering of Endoscopic Capsules by Means of a Robotic Navigation System. IEEE Transactions on Biomedical Engineering 62: 546-49

83. Martel S, Tremblay CC, Ngakeng S, Langois G. 2006. Controlled manipulation and actuation of micro-objects with magnetotactic bacteria. Applied Physics Letters 89: 233904-6

84. Tamaz S, Gourdeau R, Chanu A, Mathieu J-B, Martel S. 2008. Real-Time MRI-Based Control of a Ferromagnetic Core for Endovascular Navigation, . IEEE Trans. on Biomed. Eng. 55: 1854 - 63

85. Martel S, Mathieu J-B, Felfoul O, Chanu A, Aboussouan E, et al. 2007. Automatic navigation of an untethered device in the artery of a living animal using a conventional clinical magnetic resonance imaging system. Applied Physics Letters 90: 114105-8

86. Mathieu J-B, Martel S. 2009. Aggregation of magnetic microparticles in the context of targeted therapoes actuated by a magnetic resonance imaging system. Journal of Applied Physics 106: 044904-0044911

87. Vartholomeos P, Mavroidis C. 2010. Siimulation Platform for Self-assembly Structures in MRIguided Nanorobotic Drug Delivery Systems. In International Conference of Robotics and Automation (ICRA 2010). Anchorage, Alaska, USA: IEEE

88. Vartholomeos P, Mavroidis C, Hata N. 2010. Magnetic Targeting of Aggregated Nanoparticles for Advanced Lung Therapies: A Robotics Approach. In BIOROB 2010. Tokyo, Japan

89. Pouponneau P, Leroux J-C, Martel S. 2009. Magnetic nanoparticles encapsulated into biodegradable microparticles steered with an upgraded magnetic resonance imaging system for tumor chemoembolization. Biomaterials 
90. Sun C, Lee JSH, Zhang M. 2008. Magnetic nanoparticles in MR imaging and drug delivery. Advanced Drug Delivery Reviews 60: 1252-65

91. Seo WS, Lee JH, Sun XM, Suzuki Y, Mann D, et al. 2006. FeCo/graphitic-shell nanocrystals as advanced magnetic-resonance-imaging and near-infrared agents. Nat Mater. 5: 971-76

92. Jun YW, Huh YM, Choi JS, Lee JH, Song HT, et al. 2005. Nanoscale size effect of magnetic nanocrystals and their utilization for cancer diagnosis via magnetic resonance imaging. J Am Chem Soc 127: 5732-3

93. Lee JH, Huh YM, Jun YW, Seo JW, Jang JT, et al. 2007. Artificially engineered magnetic nanoparticles for ultra-sensitive molecular imaging. Nature: Medicine 13: 95-99

94. Xu T, Wong JT, Shikhaliev PM, Ducote JL, Al-Ghazi MS, Molloi S. 2006. Real-time tumor tracking using implanted positron emission markers: Concept and simulation study. Medical Physics 33: 2598-609

95. Park J-H, Maltzahn Gv, Zhang L, Schwartz MP, Ruoslahti E, et al. 2008. Magnetic iron oxide nanoworms for tumor targeting and imaging. Advanced Materials 20: 1630-35

96. Hamdi M, Ferreira A. 2009. Multiscale design and Modeling of Protein-based Nanomechanisms for Nanorobotics. Int. Journal of Robotics Research

97. Rotariu O, J.C. Strachan N. 2005. Modeling magnetic carrrier particle targeting in the tumor microvasculature for cancer treatment. Journal of magnetism and magnetic materials 293: pp. 63946

98. Voltairas PA, Fotiadis DI, Michalis LK. 2002. Hydrodynamics of magnetic targeting. $J$ Biomechanics 35: 813-21

99. Haverkort JW, Kenjeres S, Kleijn CR. 2009. Computational Simulations of Magnetic Particle Capture in Arterial Flows. Annals of Biomedical Engineering 37 
100. Belharet K, Folio D, Ferreira A. 2010. 3D MRI-based Predictive Control of a Ferromagnetic Microrobot Navigating in Blood Vessels. In IEEE International Conference on Biomedical Robotics and Biomechatronics (BIOROB'10)

101. Arcese L, Cherry A, Fruchard M, Ferreira A. 2010. Dynamic behavior investigation for trajectory control of a microrobot in blood vessels. In IEEE/RSJ Int. Conf. on Intel. Robots and Systems. Taipei, Taiwan

102. Arcese L, Fruchard M, Ferreira A. 2009. Nonlinear Modeling and Robust Controller-Observer for a Magnetic Microrobot in a Fluidic Environment using MRI Gradients. In Proc of the IEEE/RSJ Int. Conf. Intell. Robots Syst. St Luis, MO, USA

103. Arcese L, Cherry A, Fruchard M, Ferreira A. 2010. High gain observer for backstepping control of a MRI guided therapeutic microrobot in blood vessels. In IEEE/RAS-EMBS Int. Conf. on Biomedical Robotics and Biomechatronics (BioRob 2010)

104. Zhang L, Petit T, Lu Y, Kratochvil BE, Peyer KE, et al. 2010. Controlled Propulsion and Cargo Transport of Rotating Nickel Nanowires near a Patterned Solid Surface. ACS Nano 4 


\section{Article components}

\section{Key Words}

nanomedicine, nanorobotics, magnetic nanoparticles, MRI, targeted drug delivery, modeling, closed-loop control.

\section{Abstract}

This review paper presents the state of the art on Magnetic Resonance Imaging (MRI) guided nanorobotic systems that could perform diagnostic, curative and reconstructive treatments in the human body at the cellular and sub-cellular level in a controllable manner. The concept of a MRI-guided nanorobotic system is based on the use of a MRI scanner to induce the required external driving forces to propel magnetic nanocapsules to a specific target. It is an active targeting mechanism that provides simultaneous propulsion and imaging capabilities, which allow the implementation of real-time feedback control of the targeting process. The architecture of the system comprises four main modules: (a) the Nanocapsules, (b) the MRI Propulsion Module, (c) the MRI Tracking Module (image processing) and (d) the Controller Module. A key concept is the nanocapsule technology, which is based on carriers such as liposomes, polymer micelles, gold nanoparticles, quantum dots, metallic nanoshells and carbon nanotubes. A description is presented of the significant challenges that the MRI-guided nanorobotic system faces, and promising solutions proposed by the involved research community are discussed. Emphasis is given on reviewing the limitations impose by the scaling effects that dominate within the blood vessels and also on the review of control algorithms and computational tools that have been developed aiming at real-time propulsion and tracking of the nanocapsules. 


\section{Summary points}

- Increasing the concentration of the drug at the lesion results in significantly higher therapeutic efficiency and in minimization of the side effects at the healthy parts of the human body.

- Drug targeting mechanisms are needed to accomplish efficient control of the drug concentration at the lesion.

- The MRI-guided drug delivery is an active targeting mechanism that provides simultaneous propulsion and imaging capabilities, which allow the implementation of real-time feedback control of the targeting process.

- $\quad$ Functionalization is a key concept in nanocapsule design. Functionalized nanocapsules can efficiently target cell receptors, evade the immune system and perform controlled drug release.

- Efficient guidance of the nanocapsules requires advanced controller design and also requires the exploitation of physical phenomena such as aggregation of the magnetic nanoparticles that amplify the propulsion forces. 


\section{Future issues}

- Development of novel magnetic nanoparticles that are biocompatible, biodegradable and have improved magnetic characteristics.

- Development of MRI position detection algorithms that provide improved resolution and refresh rates.

- Development of computational tools for the systematic simulation of the dynamics of the ferrofluid, as well as for conceptualization, characterization, development and prototyping of nanocapsules. 


\section{Figure captions}

Figure 1. MRI-based nanorobotic system: a multidisciplinary field.

Figure 2. Distribution control of drug concentration.

Figure 3. MRI-guided nanorobotic system architecture.

Figure 4. Liposome formulated by phospholipids.

Figure 5. Copolymer micelle structure.

Figure 6. Silver nanoshells observed by a SEM and TEM. A) TEM image of silica sphere $1 \mu \mathrm{m}$, coated with $20 \mathrm{~nm}$ monocrystalline silver. B) SEM image of sphere from the same batch. C) TEM image of $250 \mathrm{~nm}$ sphere, with 5-10 nm silver seed decorating the surface. D) High-resolution TEM of polycrystalline silver grain. (Reproduced by permission of the Journal of Colloid and Interface Science)

Figure 7. Multi-functionalized drug loaded nanoparticle.

Figure 8. Applications of magnetic nanoparticles.

Figure 9: Variation of coercivity during transition from multidomain ferromagnetism to single domain superparamagnetism (adapted from (80)).

Figure 10. TEM images and magnetization curves of $55 \mathrm{~nm}(\mathrm{a}, \mathrm{b}) ; 12 \mathrm{~nm}(\mathrm{c}, \mathrm{d})$ (Printed by permission of American Chemical Society, (80)).

Figure 11. Aggregation of superparamagnetic particles. The particle magnitude is $5 \mu \mathrm{m}$. The aggregation shape is chain-type or clot-type and has average length $60 \mu \mathrm{m}$.

Figure 12. A) TEM image of FeCo nanoparticles. B) TEM image of the graphite shell of FeCo nanoparticles. C) SEM image of the cross-section of FeCo-PLGA microparticle. D) FeCo nanoparticle distribution in the PLGA microparticle.

Figure 13. Free body diagram of forces acting on a magnetic particle within the blood vessels.

Figure 14. Simulation of aggregations within the blood vessel. 
Figure 15: MRI-based Predictive Control strategy. 
Figures 
Figure 1

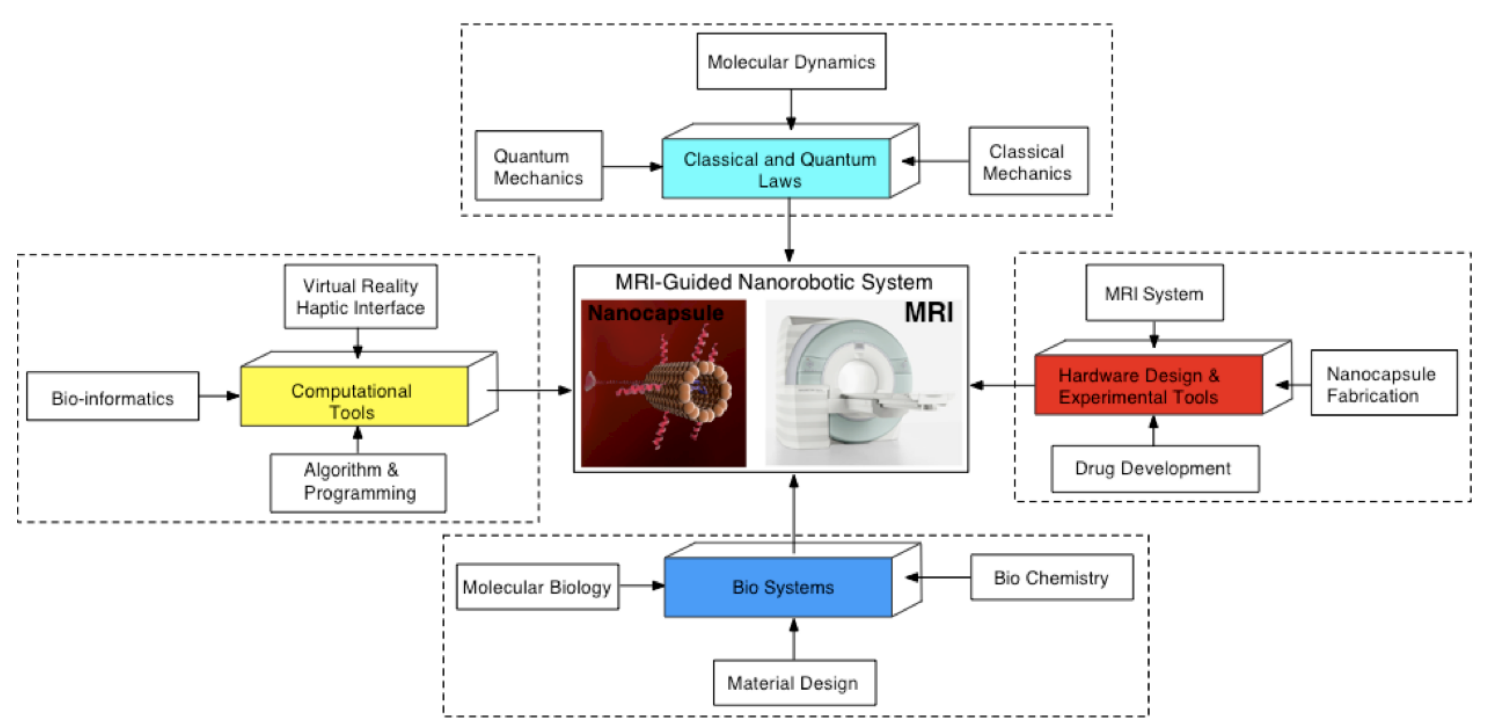


Figure 2

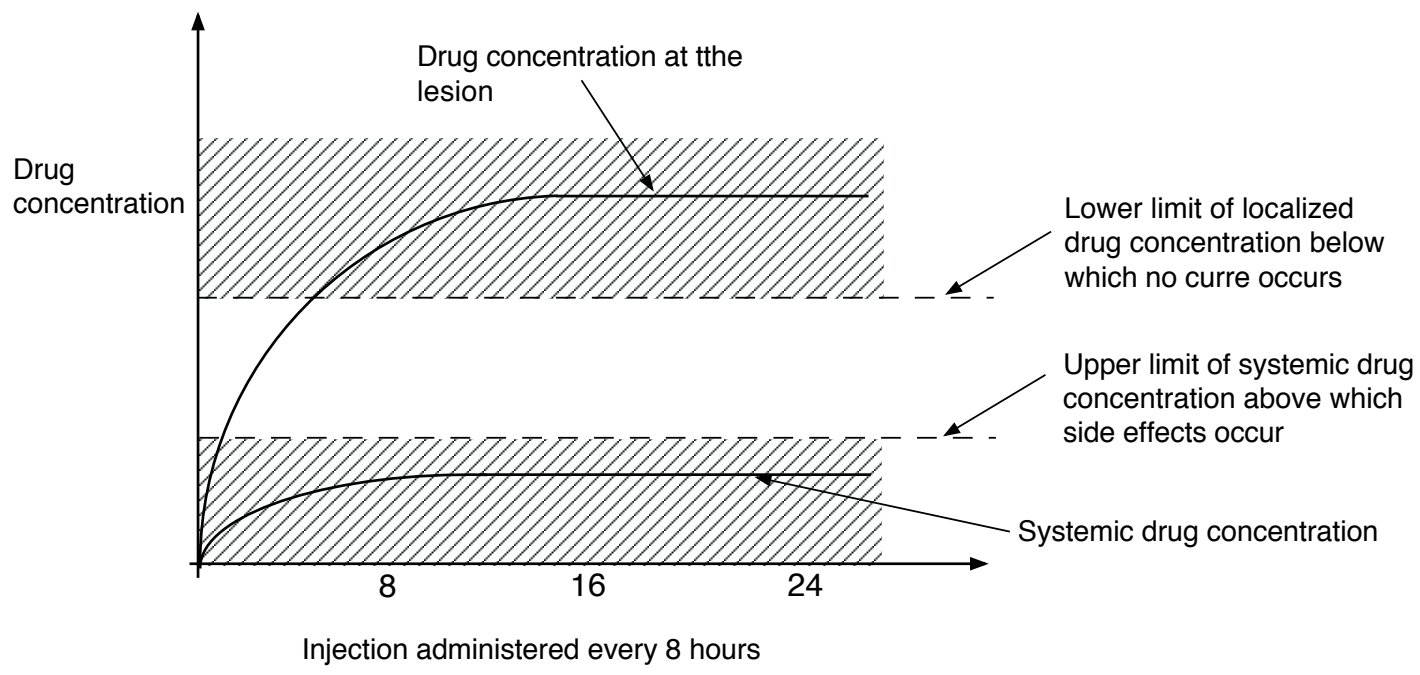


Figure 3

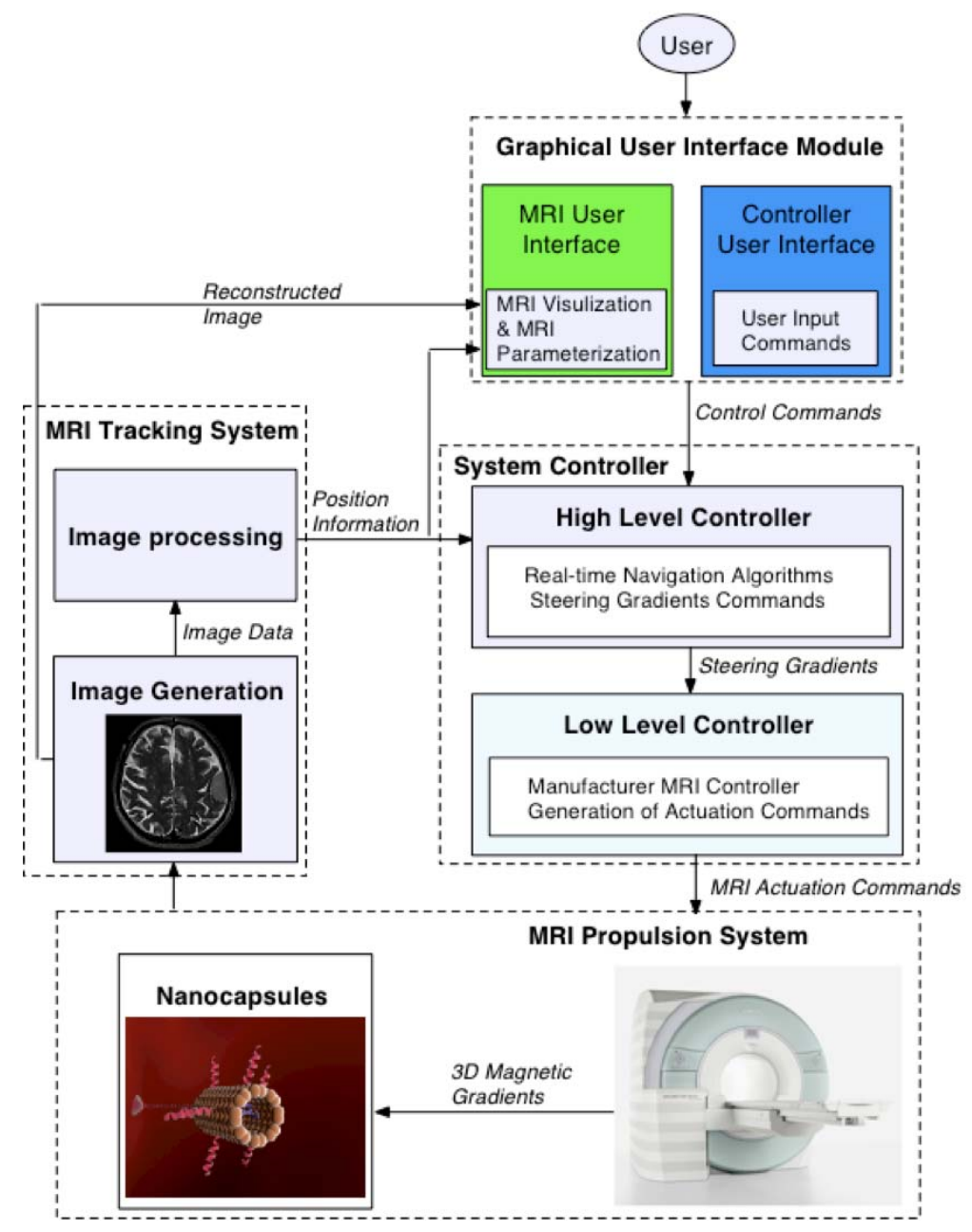


Figure 4

\section{Liposome}

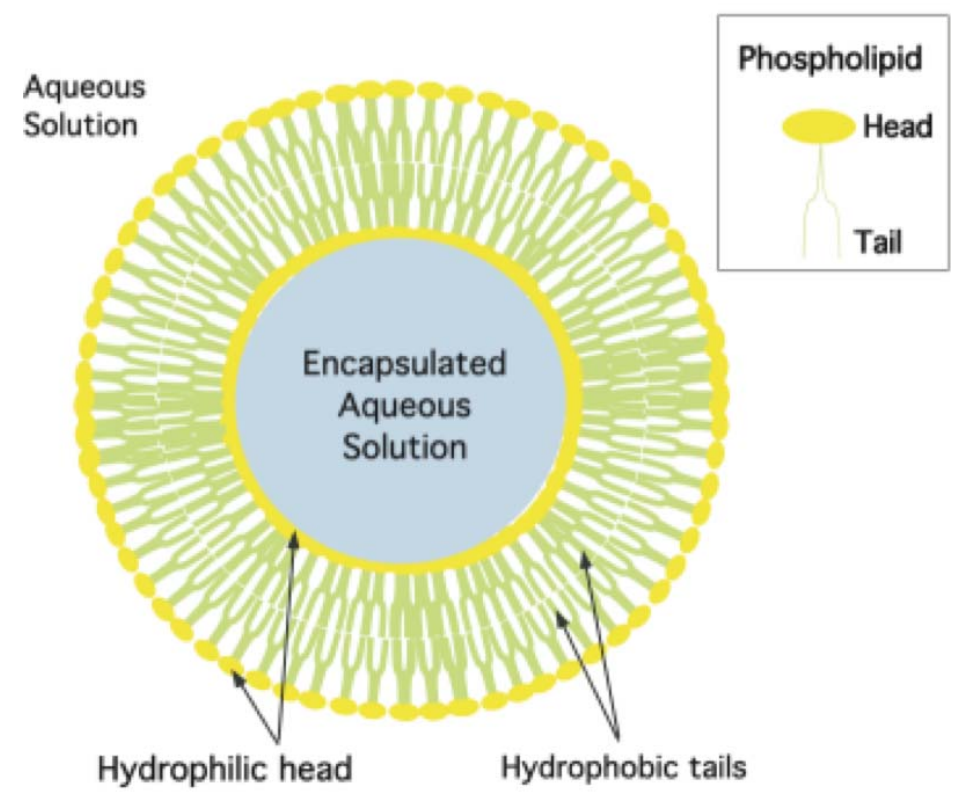


Figure 5

\section{Copolymer Micelles}

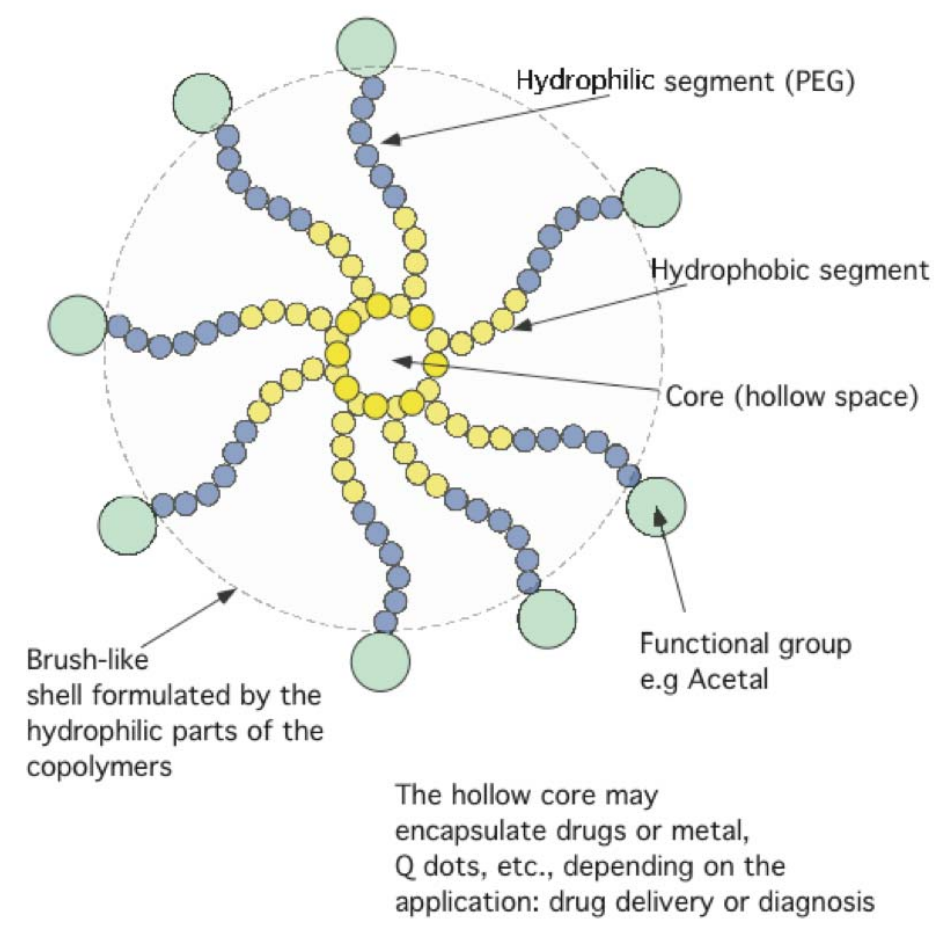


Figure 6

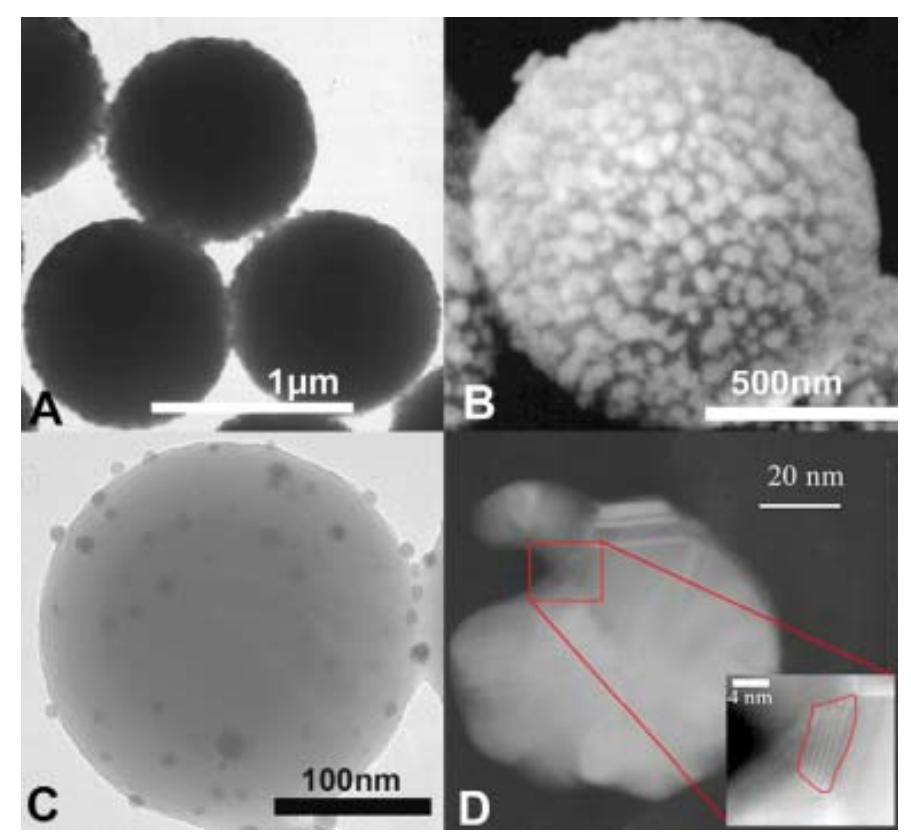


Figure 7

\section{Functionalized Nanoparticle}

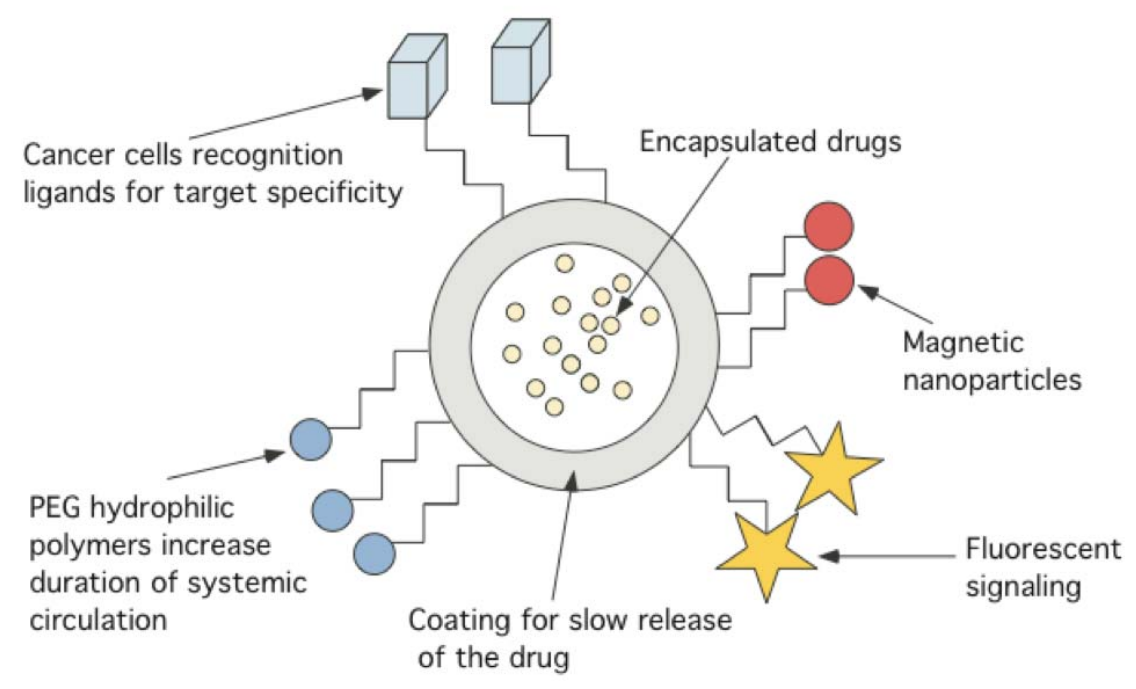


Figure 8

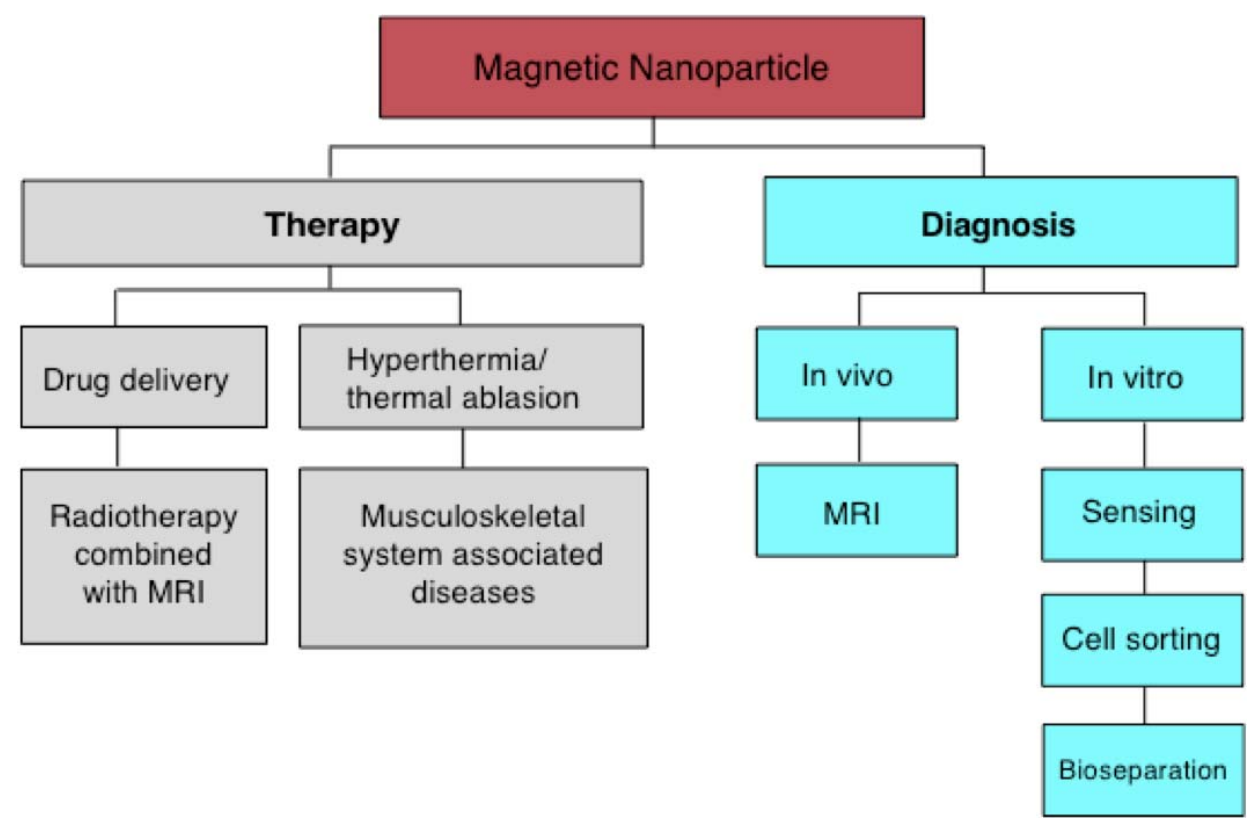


Figure 9

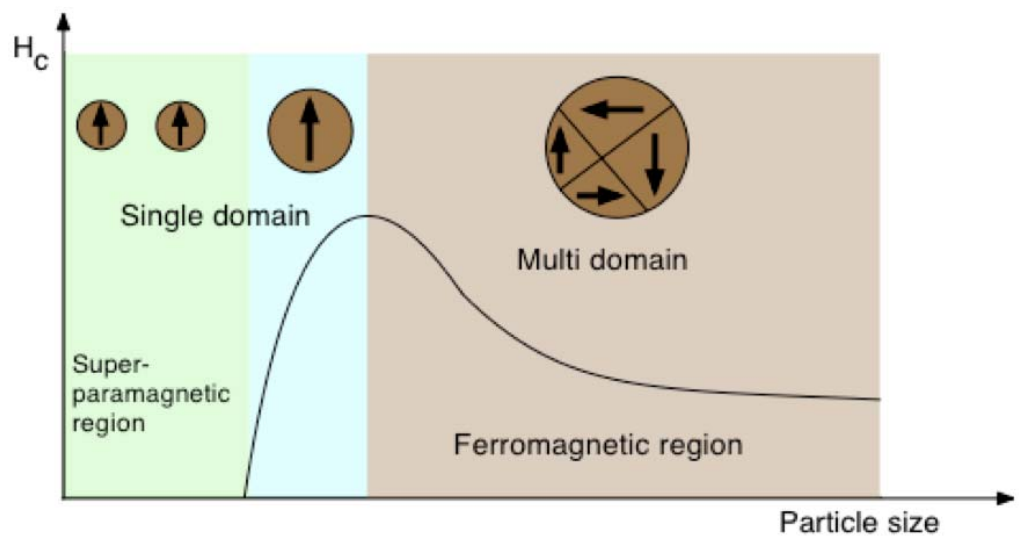


Figure 10

a)

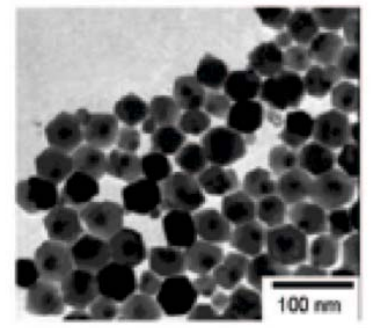

c)

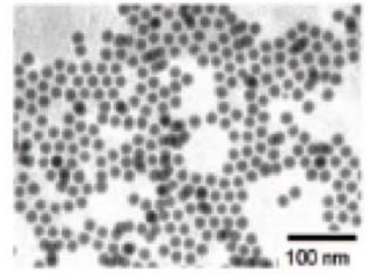

b) $\quad 0.452 \mathrm{Oe}$

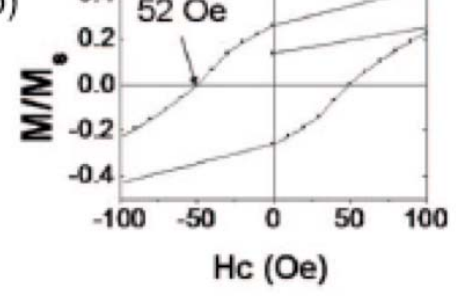

d)

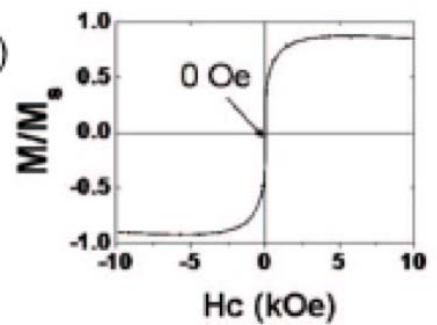


Figure 11

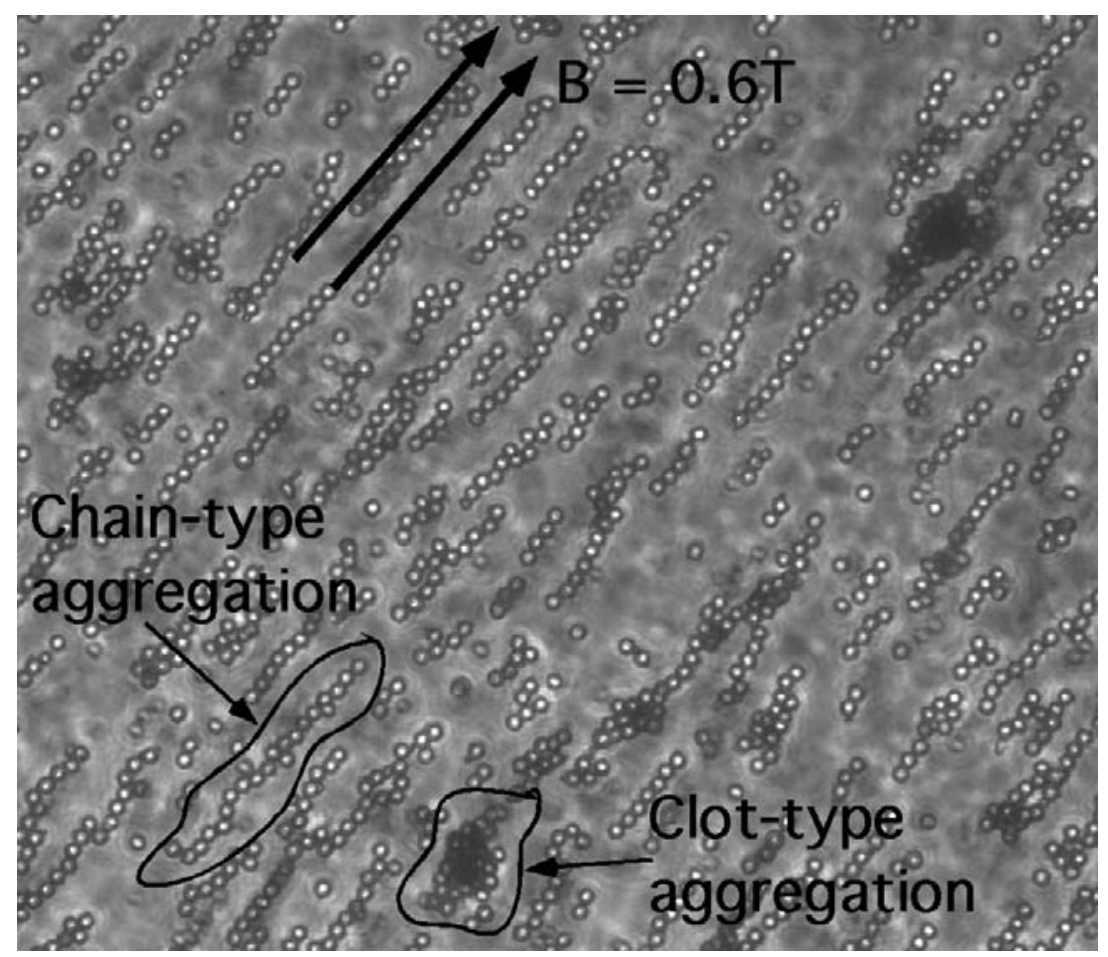


Figure 12

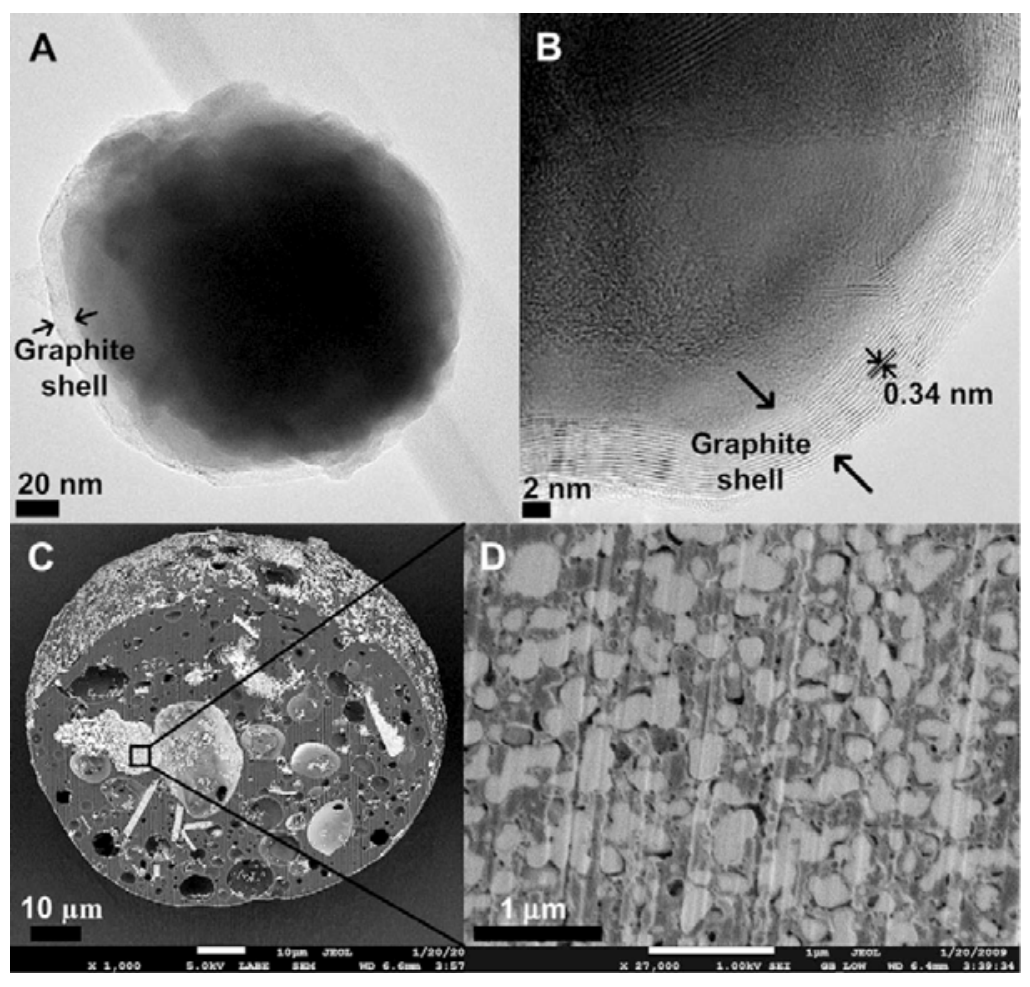


Figure 13

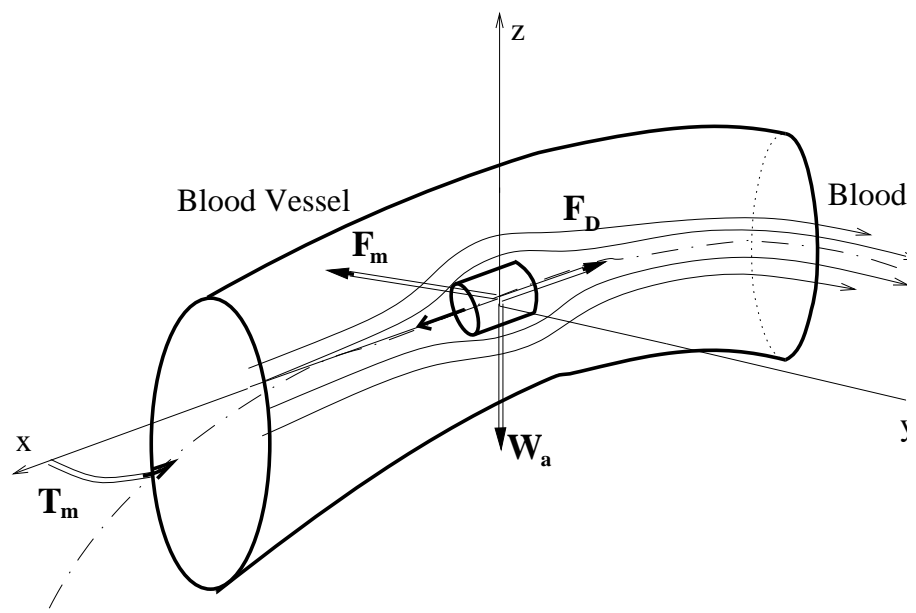

/ Trajectory 
Figure 14

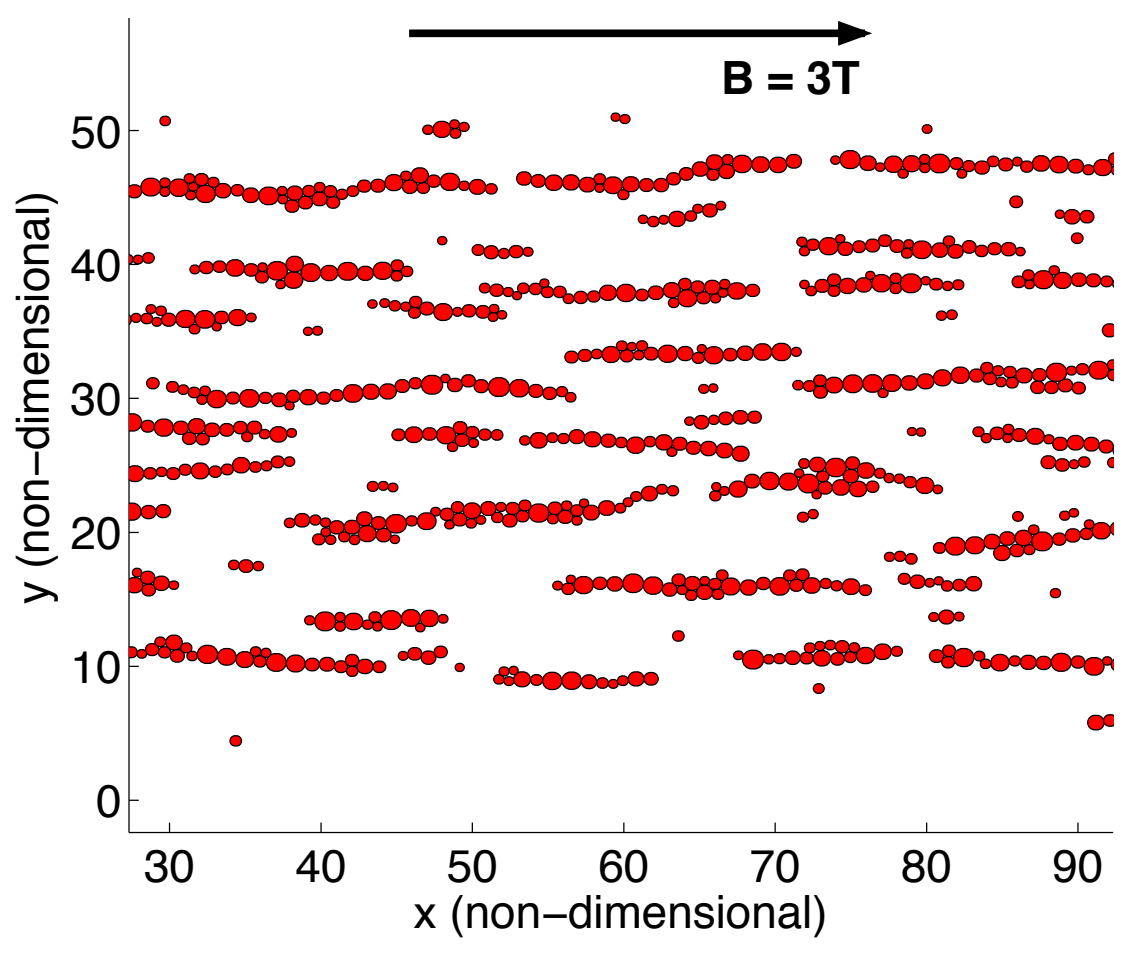


Figure 15

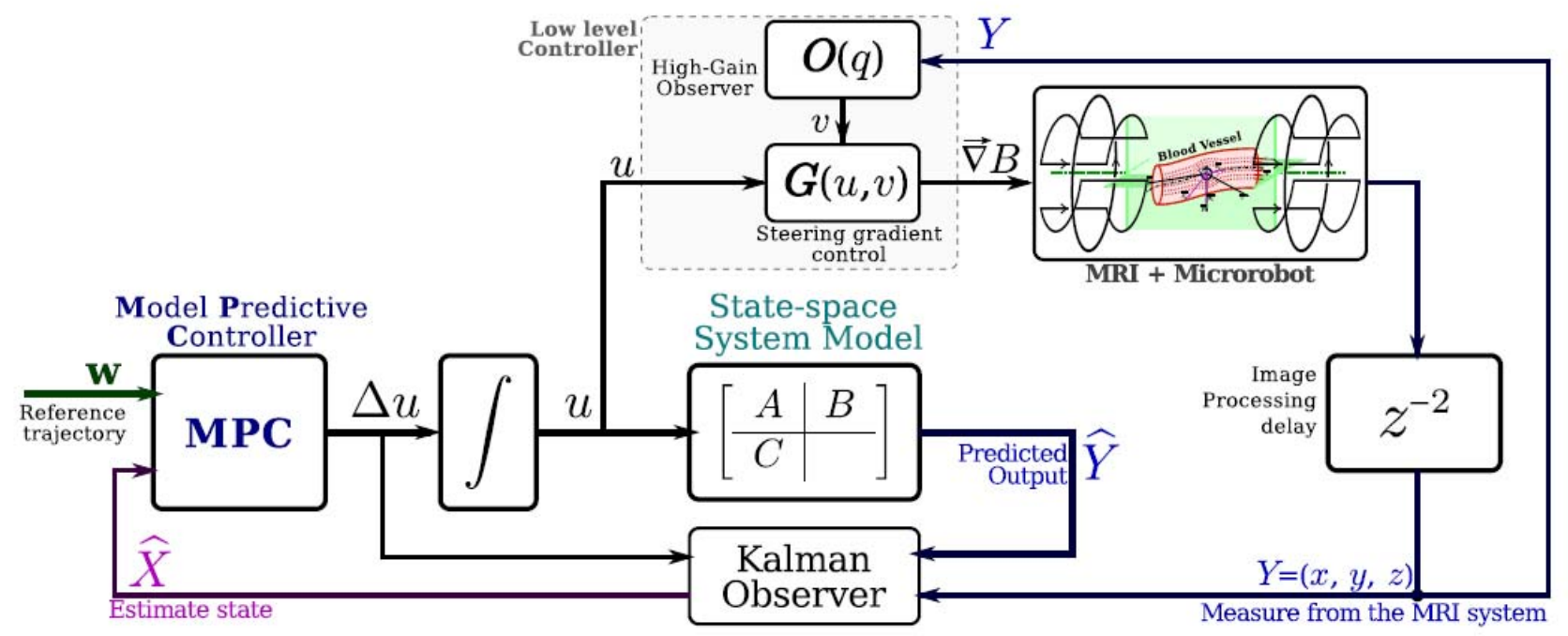

